\title{
Fundamentals of the nanowire solar cell: Optimization of the open circuit voltage
}

Cite as: Appl. Phys. Rev. 5, 031106 (2018); https://doi.org/10.1063/1.5028049

Submitted: 07 March 2018 . Accepted: 05 August 2018. Published Online: 05 September 2018

Jos E. M. Haverkort (D), Erik C. Garnett (D), and Erik P. A. M. Bakkers

\section{COLLECTIONS}

EP This paper was selected as an Editor's Pick
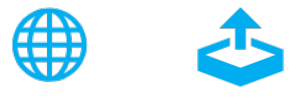

\section{ARTICLES YOU MAY BE INTERESTED IN}

Recent advances in spin-orbit torques: Moving towards device applications Applied Physics Reviews 5, 031107 (2018); https://doi.org/10.1063/1.5041793

A review on high throughput roll-to-roll manufacturing of chemical vapor deposition graphene

Applied Physics Reviews 5, 031105 (2018); https://doi.org/10.1063/1.5035295

Chip-scale atomic devices

Applied Physics Reviews 5, 031302 (2018); https://doi.org/10.1063/1.5026238 


\title{
Fundamentals of the nanowire solar cell: Optimization of the open circuit voltage
}

\author{
Jos E. M. Haverkort, ${ }^{1, a)}$ Erik C. Garnett, ${ }^{2}$ and Erik P. A. M. Bakkers ${ }^{1,3}$ \\ ${ }^{1}$ Department of Applied Physics, Eindhoven University of Technology, P.O. Box 513, 5600 MB Eindhoven, \\ The Netherlands \\ ${ }^{2}$ Center for Nanophotonics, AMOLF, Science Park 104, 1098 XG Amsterdam, The Netherlands \\ ${ }^{3}$ Kavli Institute of Nanoscience, Delft University of Technology, Lorentzweg 1, 2628 CJ Delft, The Netherlands
}

(Received 7 March 2018; accepted 5 August 2018; published online 5 September 2018)

\begin{abstract}
Present day nanowire solar cells have reached an efficiency of $17.8 \%$. Nanophotonic engineering by nanowire tapering allows for high solar light absorption. In combination with sufficiently high carrier selectivity at the contacts, the short-circuit current $\left(J_{s c}\right)$ has presently reached $29.3 \mathrm{~mA} / \mathrm{cm}^{2}$, reasonably close to the $34.6 \mathrm{~mA} / \mathrm{cm}^{2}$ theoretical limit for InP. Although further optimization of the current is important, an equally challenging condition to approach the Shockley Queisser (S-Q) limit is to increase the open-circuit voltage $\left(V_{o c}\right)$ towards the radiative limit. The key requirement to reach the radiative limit is to increase the external radiative efficiency at open-circuit conditions towards unity. It is the main purpose of this review to highlight recent progress in nanophotonic engineering to further enhance the open circuit voltage of a nanowire solar cell. In addition to material optimization for increasing the internal photoluminescence efficiency, the light extraction efficiency is a major design criterion for enhancing the external radiative efficiency and thus the $V_{o c}$. Since the semiconductor substrate is a sink for internally generated photoluminescence, it is equally important to eliminate the loss of emitted light into the substrate. Even at the S-Q limit, the $V_{o c}$ is still substantially decreased by a photon entropy loss due to the conversion of a parallel beam of photons from the sun into an isotropic emission pattern, in which each individual photon is emitted into a random direction. The $46.7 \%$ ultimate solar cell limit for direct solar irradiation can only be approached, once the cell is capable to focus all emitted photoluminescence back to the sun. We will show that nanophotonic engineering provides a pathway to approach the ultimate limit. Published by AIP Publishing.

https://doi.org/10.1063/1.5028049
\end{abstract}

\section{TABLE OF CONTENTS}

I. INTRODUCTION

II. THEORETICAL BACKGROUND ............

III. STATE-OF-THE-ART NANOWIRE SOLAR

CELLS

IV. EFFICIENT LIGHT ABSORPTION FOR A HIGH SHORT-CIRCUIT CURRENT .........

V. INTERNAL PHOTOLUMINESCENCE EFFICIENCY

VI. ENHANCED OPEN-CIRCUIT VOLTAGE BY AN ENHANCED PHOTON EXTRACTION PROBABILITY......................

VII. ENHANCEMENT OF THE $\mathrm{V}_{\mathrm{OC}}$ BY A REDUCED EMISSION INTO THE

SUBSTRATE .........................
VIII. TOWARDS THE ULTIMATE EFFICIENCY SOLAR CELL .......................

IX. ECONOMICS OF NANOWIRE SOLAR

CELLS ..............................

$\mathrm{X}$. CONCLUSIONS ........................

a) Author to whom correspondence should be addressed:
J.E.M.Haverkort@tue.nl

\section{INTRODUCTION}

In this review, we will discuss several options to increase the open-circuit voltage in a solar cell, with a special emphasis towards nanowire solar cells. Most of the literature on solar cells, including several reviews ${ }^{1-9}$ on nanowire solar cells, are mainly focused towards increasing the short-circuit current while not discussing the opencircuit voltage in much detail. The open-circuit voltage can be understood by considering the detailed balance limit of a solar cell which was developed by Shockley and Queisser ${ }^{10}$ and which has been re-visited and put into historical perspective by Queisser ${ }^{11}$ in 2009 . The essential ingredients are that the maximum achievable carrier lifetime in a semiconductor is ultimately limited by the 
radiative recombination lifetime. In addition, the minimum achievable recombination current is ultimately limited by the black-body recombination current. We emphasize that both limits are radiative quantities. The detailed balance principle subsequently connects the black-body emission rate with the electron-hole generation rate excited by thermal radiation in the dark. For a lossless cell under solar irradiation, detailed balance requires that the external radiative recombination rate equals the solar generation rate. These simple principles determine the maximum opencircuit voltage of any solar cell. Achieving a high open circuit voltage thus requires an optimization of the external radiative efficiency, as will be discussed in Sec. II.

It is well known that every type of solar cell suffers from a so-called $V_{o c}$-deficit, which is the difference between the measured $V_{o c}$ and the material's bandgap. The $V_{o c}$-deficit has been reviewed in recent solar cell papers by Polman et al. ${ }^{12}$ and Green and Bremner. ${ }^{13}$ Knowledge on the precise steps required to increase the $V_{o c}$ up to the materials bandgap is not widespread. In this review, we will show that although the $V_{o c}$-deficit is strongly influenced by the internal photoluminescence efficiency, the $V_{o c}$-deficit is also strongly determined by the (nano)photonic design of the solar cell. We emphasize that these nanophotonic design principles apply to any type of solar cell and are thus generic. III-V semiconductor nanowire solar cells provide a workhorse to optimize and to study these nanophotonic solar cell properties.

Recently, a lot of progress was made in the field of nanowire solar cells. Important milestones were the $13.8 \%$ efficiency InP nanowire solar cell ${ }^{14}$ reported by Lund, the $15.3 \%$ efficiency GaAs nanowire solar cell ${ }^{15}$ reported by Solvoltaics in Lund, and finally the $17.8 \%$ efficiency InP nanowire solar cell ${ }^{16}$ reported by us. The state-of-the-art in nanowire solar cells is discussed in Sec. III.
In this review, we will focus on increasing the $V_{o c}$. We, however, start with a discussion on the light absorption in nanowire arrays. Nanophotonic optimization of the light absorption in the present $17.8 \%$ efficiency nanowire solar cell has increased the measured $J_{s c}$ reasonably close to the limit $^{17}$ for InP. The intrinsic advantage of nanowire solar cells for current optimization will be discussed in Sec. IV.

Optimization of the open-circuit voltage is the main challenge in nanowire solar cell research. It is the prime focus of this review. Approaching a $V_{o c}$ close to the materials bandgap requires a 3-stage approach. In the first stage, the internal photoluminescence efficiency should be optimized towards $100 \%$. This optimization requires that the radiative recombination rate is significantly larger than the nonradiative recombination rate, which hitherto has mainly been demonstrated for high quality planar GaAs. Optimization of the internal photoluminescence efficiency will be discussed in Sec. V.

In order to approach the radiative limit, it is equally important to optimize the photon extraction probability at the front-side of the solar cell, since thermodynamics requires that the external radiative efficiency should be close to unity for approaching the Shockley Queisser (S-Q) limit ${ }^{10}$ (see Box 1). Optimization of the photon escape probability will be discussed in Sec. VI.

At this point, the radiative losses into the semiconductor substrate should be eliminated. A semiconductor substrate features a high photonic density of states, which acts as an efficient sink for internally generated photoluminescence within the solar cell. Even a planar III/V semiconductor solar cell can only reach the limiting S-Q efficiency when radiative losses into the substrate are eliminated. The objective is that the front-side emission equals the solar radiation flux at open-circuit conditions. Substrate losses will be further discussed in Sec. VII.

\section{The Shockley-Queisser and the ultimate limit}

The Shockley-Queisser limit and the ultimate limit for direct solar radiation are both plotted in Fig. 1. While the S-Q limit peaks at $33.7 \%$ near a bandgap of $1.34 \mathrm{eV}$, the ultimate efficiency for direct solar radiation shows two maxima at $46.6 \%(0.95 \mathrm{eV})$ and $46.7 \%(1.13 \mathrm{eV})$, respectively. Since the light entropy contribution always increases the $V_{o c}$ with approximately $0.3 \mathrm{~V}$, the ultimate efficiency is more strongly increased at lower bandgaps. A low bandgap cell is harvesting a larger portion of the solar spectrum and is thus generating a larger current. The current should be multiplied by the $0.3 \mathrm{~V}$ increase in $V_{o c}$ due to the light entropy contribution, yielding the largest increase in the efficiency for a low bandgap cell. The ultimate efficiency is still limited by losses due to (1) solar photons which are not absorbed since they have an energy below the semiconductor bandgap, (2) thermalization loss of photoinduced carriers by photons with a photon energy above the bandgap; the excess energy can only be converted into heat, (3) the Carnot efficiency, (4) a loss connected to the fact that the voltage at the maximum power point of a solar cell is smaller than the open circuit voltage, and (5) a loss due to emission of photons at the maximum power point of the solar cell.

For terrestrial conditions, the amount of direct $\left(0.5^{\circ}\right.$ solar cone $)$, circumsolar $\left( \pm 2.5^{\circ}\right.$ solar cone $)$, and diffuse solar radiation depend on the weather conditions. In the American Society for Testing and Materials (ASTM) G173-03 spectrum, $90 \%$ of the solar light is direct and can be converted with the ultimate efficiency, while $10 \%$ is diffuse. When all emitted light is sent back to the sun, reciprocity of light propagation dictates that diffuse light cannot be absorbed within the spectral region of the emission spectrum. However, it is possible that diffuse light outside this spectral region can be absorbed by the cell and subsequently can be converted with the S-Q efficiency, implying a limiting efficiency of $\approx 44 \%$. A detailed analysis of light absorption and emission in an ultimate limit solar cell, taking into account light scattering in the atmosphere, would be an important and interesting direction for future research. 


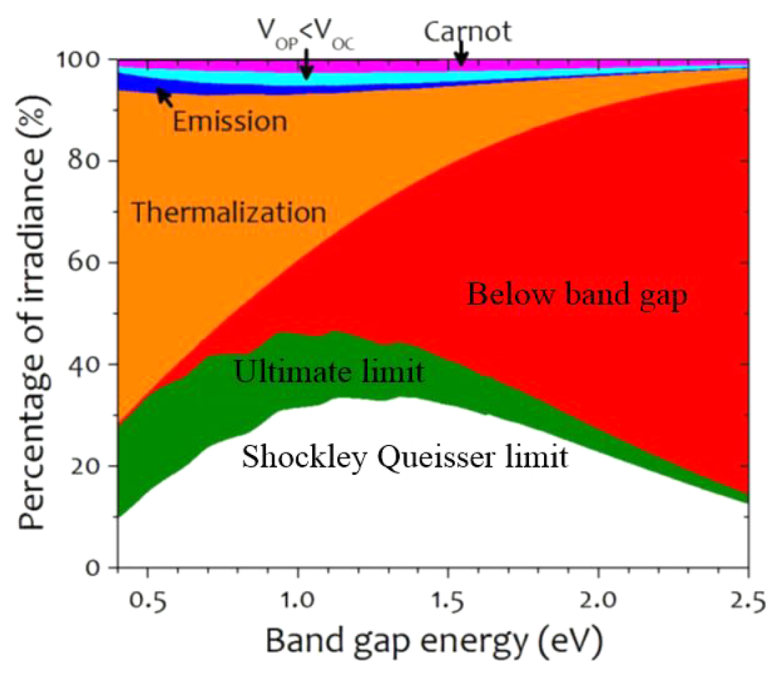

FIG. 1. Plot of the ultimate efficiency for direct solar radiation as well as the Shockley Queisser limit versus the semiconductor bandgap based on the Air Mass 1.5 Global Spectrum (ASTM173-03 G).

Once the S-Q limit is reached, it is important to realize that the solar cell is converting a nearly parallel beam of photons (assuming direct radiation) originating from the sun into a randomized emission pattern when it is operating at open circuit conditions. The solar cell is thus strongly increasing the entropy of the impinging solar light, which translates into a reduction of the open circuit voltage by ${ }^{18} \approx 0.3 \mathrm{~V}$ for direct solar radiation. This voltage loss can only be eliminated by sending all emitted photons back towards the sun, resulting in an ultimate limit solar cell (see Box 1). The ultimate limit was first proposed by Shockley and Queisser ${ }^{10}$ when assuming a fictitious sun illuminating the solar cells with a $4 \pi$ input solid angle. In this way, Shockley and Queisser obtained an ultimate efficiency $^{10}$ of $\approx 43 \%$ by assuming a simplified black-body radiation spectrum. The $V_{o c}$-deficit can only be completely eliminated in an ultimate limit solar cell. The light propagation in an ultimate limit cell should be strictly reversible within the spectral region of the emission spectrum, thus avoiding any increase in photon entropy. The ultimate limit will be discussed in Sec. VIII. Finally, we shortly discuss the economics of nanowire photovoltaic (PV) as well as recent efforts towards nanowire tandem cells in Sec. IX.

Although it is most appealing to present this approach as a three step approach, it is possible to increase the solar cell efficiency by nanophotonic engineering in which one is starting with a lower internal photoluminescence efficiency or a lower photon extraction probability. We also like to stress that this review is academic in the sense that most of the steps required to reach a high $V_{o c}$ have never been tested under realistic outdoor conditions. However, we will use the AM1.5G solar spectrum, which is corrected for, e.g., water absorption and light scattering in the atmosphere, when calculating the limiting efficiencies. In addition, most of the cited work has been performed for idealized solar radiation at normal incidence. We will, however, discuss the dependence on the angle of incidence for a nanowire solar cell in Sec. IV.

\section{THEORETICAL BACKGROUND}

A solar cell is characterized by its short-circuit current, its open-circuit voltage, and its fill factor. It is well known that the short-circuit current of a nanostructured solar cell is determined by the absorptance of the solar light as well as by the efficiency for charge separation and collection. These quantities are characterized by the internal quantum efficiency (IQE) for the conversion of an absorbed photon into current and the external quantum efficiency (EQE) which characterizes the conversion efficiency of incident solar photons into current. A nanostructure is capable to convert incident photons which are impinging in close proximity to its geometrical cross section due to the wave character of light. One thus needs to consider the absorption cross section $\sigma_{a b s}$ instead of its geometrical cross section ${ }^{20}$ for obtaining the short-circuit current. For a vertically standing nanowire, the absorption cross section is both wavelength and diameter dependent. The Finite Difference Time Domain (FDTD) simulations ${ }^{19}$ presented in Fig. 2 show a maximum absorption cross section which is at least $12 \times$ larger than the geometrical cross section of the nanowire, allowing to also harvest many solar photons impinging "in-between" different nanowires within an array.

For a single nanowire solar cell, the EQE is proportional to the absorption cross section of the nanowire. As an example, for a horizontally oriented single nanowire, the EQE cross section can be written as ${ }^{21}$

$$
\sigma_{E Q E}(\lambda, \Omega)=\int \operatorname{IQE}(\lambda, z) \sigma_{a b s}^{1 D}(\lambda, \Omega) d z
$$

in which $\sigma_{a b s}^{1 D}(\lambda, \Omega)$ is the effective absorption width of the nanowire. In the case of a nanowire array solar cell, the idea is to position the vertically oriented nanowires close enough together to make sure that the absorption cross sections of the individual nanowires start to overlap each other. The light absorption in nanowire solar cells will be further discussed in Sec. IV.

As compared to the amount of literature on the optimization of the short-circuit current, the mechanisms that determine the open-circuit voltage $\left(V_{o c}\right)$ are less well-known. In this review, we will therefore focus on the open-circuit voltage $\left(V_{o c}\right)$.

Imagine that a solar cell is irradiated by solar photons with a solar generation rate $R_{\text {sun }}$ at $\mathrm{AM} 1.5 \mathrm{G}$ solar conditions. At the open-circuit voltage, these photons are not contributing to the current, which is by definition zero. So, any absorbed solar photon at $V=V_{o c}$ has only two options; it either is converted into heat in a nonradiative recombination process or is externally re-emitted. In a lossless solar cell at $V=V_{o c}$, the only possibility is to externally re-emit all absorbed solar photons. ${ }^{22}$ The external radiative recombination rate, $R_{e x t}$, should thus be exactly equal to the solar generation rate $R_{\text {ext }}=R_{\text {sun }}$. This implies that the external radiative efficiency $\eta_{e x t}^{P L}=\frac{R_{e x t}}{R_{e x t}+R_{\text {rrad }}}$ provides a direct metric ${ }^{13}$ for the ability of a solar cell to approach the radiative limit $V_{o c}^{r a d}$. At the Shockley Queisser limiting efficiency of $33.7 \%$ at $\mathrm{AM} 1.5 \mathrm{G}$ conditions, the open-circuit voltage is equal to 


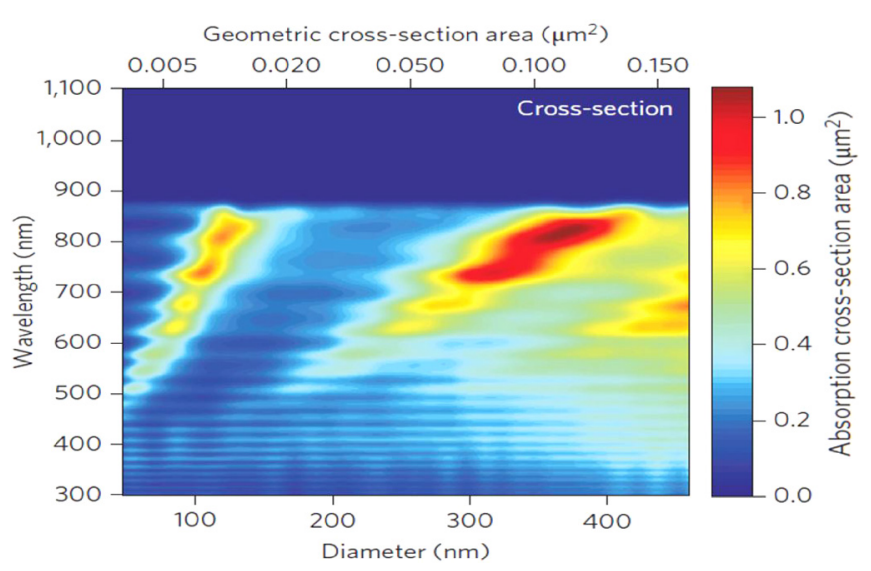

FIG. 2. Finite difference time domain (FDTD) simulation of the absorption cross section $^{19}$ for a $2.5 \mu \mathrm{m}$ standing GaAs nanowire embedded in a medium with refractive index $n=1.67$ on a silicon substrate for plane-wave illumination propagating along the nanowire axis. It is important to note that the absorption cross section is considerably larger than the geometrical cross section. Reproduced with permission from Krogstrup et al., Nat. Photonics 7, 306-310 (2013). Copyright 2013 Springer Nature.

the radiative limit, while the short-circuit current and the fill factor should also be fully optimized. The experimental relation between $\eta_{\text {ext }}^{P L}$ and the solar cell efficiency is highlighted in Fig. 3, showing a clear relation between the power conversion efficiency and the logarithm of the external radiative efficiency. The red dashed line represents the efficiency for optimum light absorption, current conversion, and fill factor for an optimum bandgap solar cell which is only limited by the external radiative efficiency [see Eq. (8)]. It can be seen that cells with a large $V_{o c}$-deficit also suffer additional losses due to incomplete current conversion and/or a reduced fill factor. To reach the Shockley Queisser limit, it is required that both $\eta_{\text {ext }}^{P L}=1$ and $E Q E=1$. In addition, resistive losses

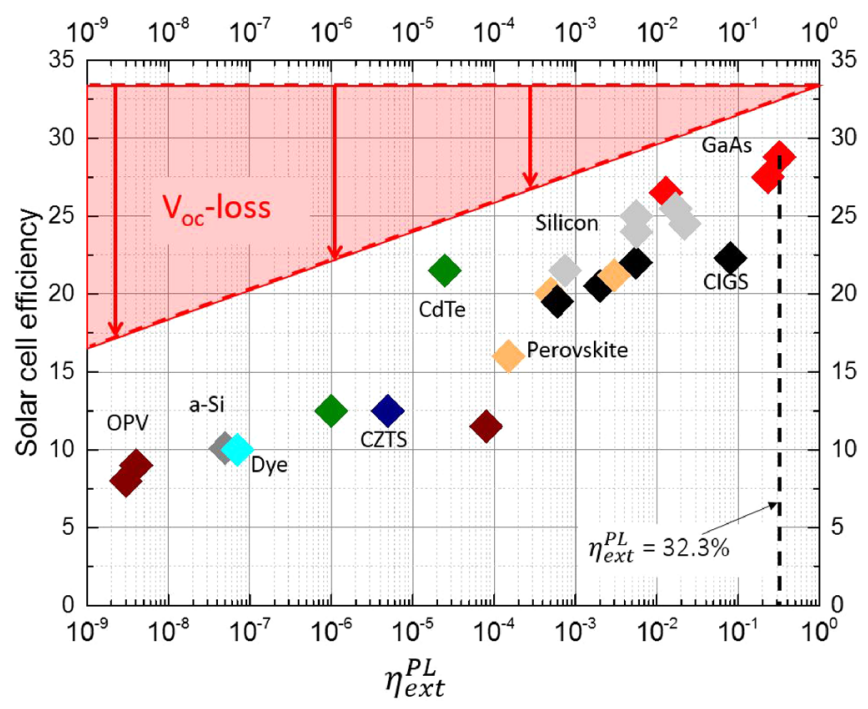

FIG. 3. Solar energy conversion efficiency versus the external radiative efficiency for different types of solar cells. The red triangle indicates the $\mathrm{V}_{\mathrm{oc}}$-loss as calculated with Eq. (8) and allows to distinguish the $\mathrm{V}_{\mathrm{oc}}$-loss from other loss mechanisms in each cell. The record GaAs cell from Alta Devices has an external radiative efficiency of $32.3 \%$ and a power conversion efficiency of $28.8 \%$. Reproduced and modified with permission from M. A. Green and S. P. Bremner, Nat. Mater. 16, 23-34 (2016). Copyright 2016 Springer Nature. should be absent. The highest internal radiative efficiency ever reported ${ }^{23}$ is $99.7 \% \pm 0.2 \%$ in an $\mathrm{AlGaAs} / \mathrm{GaAs} /$ AlGaAs double heterostructure in which surface recombination is virtually absent, corresponding to an external radiative efficiency of $72 \%$ without using an anti-reflection coating. For InP, a high internal radiative efficiency of $97 \%$ has been reported. ${ }^{24}$ The highest external radiative efficiency reported in a solar cell is $32.3 \%$ for the record GaAs solar cell $^{13}$ and $20.8 \%$ in a record GaInP cell. ${ }^{25}$

Kirchhoff's radiation law requires that the emissivity of a body is equal to its absorptance. ${ }^{18}$ The absorptance by black-body radiation is equal to $a(E) \varphi_{\text {Black-body }}(T, E)$ in which $\varphi_{\text {Black-body }}(T, E)$ is the black body's photon flux per unit area at cell temperature $\mathrm{T}$ and $a(E)$ is the dimensionless absorbance for a solar photon of energy $E$. In a welldesigned solar cell, the absorbance $a(E)$ is zero below the bandgap and should be close to unity above the bandgap. According to Kirchhoff's law, the flux of emitted solar photons is equal to $a(E) \varphi_{\text {Black-body }}(T, E)$. For simplicity, $a(E)$ is taken to be independent of the incident angle of the solar photon. (It is, however, straightforward to include the incident angle dependence by replacing $a(E)$ by $a(E, \theta, \varphi)$ as well an integration ${ }^{22,26}$ over $\theta$ and $\phi$.) In the dark, the solar cell will have the same temperature $\mathrm{T}$ as the surrounding thermal bath. For an ideal solar cell in the dark, Kirchhoff's radiation law thus requires that the rate of black-body recombination per frequency interval is equal to the generation rate of electron-hole pairs by the thermal radiation, which implies ${ }^{18,22}$

$$
R_{\text {Black-body }}=\varepsilon_{\text {out }} \int a(E) \varphi_{\text {Black-body }}(T, E) d E,
$$

where $\varepsilon_{\text {out }}$ is the étendue of the emitted light. For a nanostructured cell, this equation simply yields the black body radiation $R_{\text {black-body }}$ emitted by the flat-plate area of the solar cell at room temperature. Under solar radiation, the cell is no longer in equilibrium. The solar radiation is continuously generating a quasi-Fermi level splitting or internal chemical potential $\mu$ within the cell. For a lossless cell (no nonradiative recombination and perfect contacts), the radiative recombination ${ }^{10}$ will increase proportional to $\frac{n p}{n^{2}}$, which is equal to $e^{\mu / k T}$ according to the mass-action law. As a consequence, the external radiative recombination becomes exponentially dependent on the quasi-Fermi level splitting $\mu$ according to

$$
R_{\text {ext }}(\mu)=\varepsilon_{\text {out }} \exp \left(\frac{\mu}{k_{B} T}\right) \int a(E) \varphi_{\text {Black-body }}(T, E) d E .
$$

In the absence of series resistances due to, e.g., the metalsemiconductor contacts, the voltage $V$ across the solar cell is directly related to the quasi Fermi level splitting $\mu=q V$, yielding 20,27

$$
R_{\text {ext }}(V)=\exp \left(\frac{q V}{k_{B} T}\right) R_{\text {black-body }}
$$

which is the equation for the light emission from a Light Emitting Diode (LED). For a lossless solar cell, it is 
instructive to note that the I-V curve under solar illumination can be interpreted as a black-body emitter at $\mathrm{V}=0$, which increases with a factor $\exp \left(\frac{q V}{k_{B} T}\right)$ until the external radiative emission rate is equal to the solar generation rate at $V=V_{o c}$, as is illustrated in Fig. 4.

The solar generation rate can be written as ${ }^{18}$

$$
R_{\text {sun }}=\varepsilon_{\text {in }} \int a(E) \varphi_{\text {sun }}(E) d E
$$

where $\varepsilon_{i n}$ is the étendue of the incoming solar light and $\varphi_{\text {sun }}(E)$ is the incident solar flux. In this review, the incident solar flux is calculated with the AM1.5G solar spectrum which includes atmospheric effects such as atmospheric transmission losses due to water absorption and light scattering. Unless stated otherwise, we take the axis of the solar cone at normal incidence. The étendue of the incoming solar light is, however, simplified by assuming direct solar irradiation. The effect of atmospheric scattering on the ultimate limit is a subject for future research. The detailed balance conditions $^{11,28}$ for an ideal cell require that at $V=V_{o c}$, $R_{\text {ext }}=R_{\text {sun }}$, yielding the radiative limit $V_{o c}^{\text {rad }}\left(\frac{\varepsilon_{\text {in }}}{\varepsilon_{\text {out }}}\right)$, which is still a function of the ratio of the input and output étendue $\frac{\varepsilon_{\text {in }}}{\varepsilon_{\text {out }}}$

$$
V_{o c}^{\text {rad }}\left(\frac{\varepsilon_{\text {in }}}{\varepsilon_{\text {out }}}\right)=\frac{k_{B} T}{q} \ln \frac{\varepsilon_{\text {in }} \int a(E) \varphi_{\text {sun }}(E) d E}{\varepsilon_{\text {out }} \int a(E) \varphi_{\text {Black-body }} d E}=\frac{k_{B} T}{q} \ln \frac{J_{s c}}{J_{0}}
$$

This relation directly provides the well-known relation for the open circuit voltage of an ideal cell in terms of the ratio of the short-circuit current $J_{s c}$ and the recombination current $J_{0}$. It should be emphasized that for a lossless cell, the

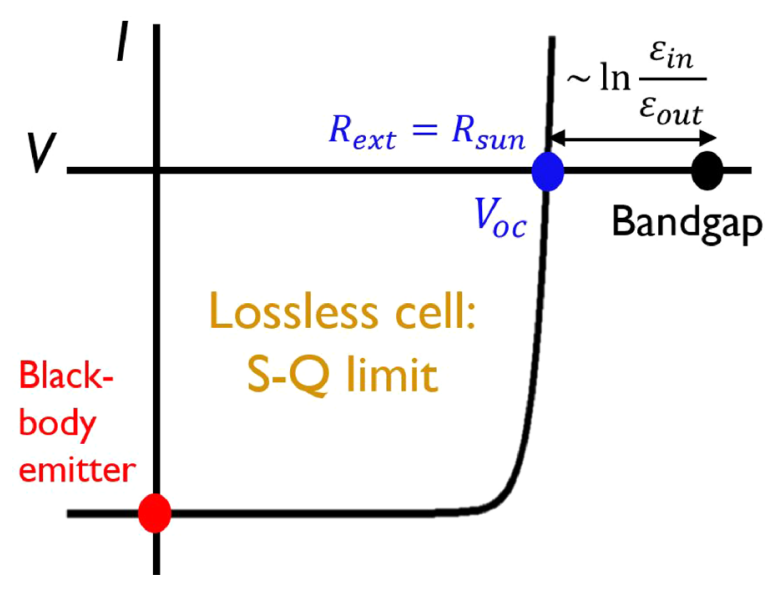

FIG. 4. Current-voltage characteristics of a solar cell emphasizing the similarity between a solar cell and a light-emitting diode (LED). At V=0, the cell converts all solar radiation into current but is only emitting black-body radiation. At $\mathrm{V}=\mathrm{V}_{\mathrm{oc}}$, the external radiative emission becomes equal to the solar generation rate for a lossless cell at AM1.5G solar irradiation. Above $\mathrm{V}_{\mathrm{oc}}$, the cell generates more light than it absorbs and is acting like a LED. For direct solar irradiation, the difference between the open circuit voltage at the Shockley-Queisser limit and the semiconductor bandgap is mainly determined (see Sec. VIII) by the solid angle of the incident solar light divided by the solid angle of the emitted photoluminescence. recombination current is only determined by black-body recombination. In the $\mathrm{S}-\mathrm{Q}$ limit, this relation reduces to the radiative limit for the open-circuit current, which reads $V_{o c}^{r a d}\left(\frac{\varepsilon_{\text {in }}}{\varepsilon_{\text {out }}}=\frac{6.85 \times 10^{-5}}{4 \pi}\right)=V_{o c}^{r a d}=V_{o c}^{S Q}$. The radiative limit $V_{o c}^{r a d}$ is lower than the ultimate short-circuit voltage $V_{o c}^{\text {rad }}\left(\frac{\varepsilon_{\text {in }}}{\varepsilon_{\text {out }}}=1\right)=V_{o c}^{\text {Ultimate }}$. Both the S-Q limit and the ultimate limit were first introduced in the seminal paper of Shockley and Queisser and are related by

$$
V_{o c}^{\text {rad }}=V_{o c}^{\text {Ultimate }}-\left|\frac{k_{B} T}{q} \ln \frac{\varepsilon_{\text {in }}}{\varepsilon_{\text {out }}}\right| .
$$

The ultimate open circuit voltage is very close to the semiconductor bandgap. The small difference between these two quantities will be further discussed in Sec. VIII. We have assumed an ideal cell until this point. For a non-ideal solar cell, nonradiative recombination reduces the external radiative recombination rate by the external luminescence efficiency. At open circuit conditions, detailed balance in the presence of nonradiative recombination now requires $R_{\text {ext }}=R_{\text {sun }} \eta_{\text {ext }}^{P L}$, yielding $^{18,29}$

$$
\begin{aligned}
V_{o c} & =V_{o c}^{\text {Ultimate }}-\left|\frac{k_{B} T}{q} \ln \frac{\varepsilon_{\text {in }}}{\varepsilon_{\text {out }}}\right|-\left|\frac{k T}{q} \ln \left(\eta_{\text {ext }}^{P L}\right)\right| \\
& =V_{o c}^{\text {rad }}-\left|\frac{k T}{q} \ln \left(\eta_{\text {ext }}^{P L}\right)\right| .
\end{aligned}
$$

This equation, which still describes a cell with perfect contacts, was first derived by Ross. ${ }^{30}$ An equivalent relation was derived by Smestad and Ries ${ }^{31}$ and is called a reciprocity relation. ${ }^{32,33}$ This equation directly shows the counterintuitive fact that one should optimize the external photoluminescence efficiency towards unity ${ }^{18,22,29,34}$ to obtain the radiative limit, which is, in turn, a necessary condition to reach the Shockley Queisser limit. The efficiency for the external emission of internally generated photoluminescence, $\eta_{\text {ext }}^{P L}$, can be separated into the internal radiative efficiency $\eta_{i n t}^{P L}$ and an angle-averaged light extraction efficiency, $\overline{P_{\text {esc }}}$, by $^{34,35}$

$$
\eta_{\text {ext }}^{P L}=\frac{\eta_{\text {int }}^{P L} \overline{P_{e s c}}}{1-\eta_{\text {int }}^{P L}\left(1-\overline{P_{e s c}}\right)} \approx \eta_{\text {int }}^{P L} \overline{P_{e s c}}=\frac{\tau_{\text {rad }}^{-1}}{\tau_{\text {rad }}^{-1}+\tau_{\text {nrad }}^{-1}} \overline{P_{e s c}},
$$

where $\tau_{\text {rad }}$ and $\tau_{\text {nrad }}$ are the radiative and the nonradiative recombination lifetimes, respectively.

These results show that it is a first task to optimize the radiative recombination rate $\tau_{\text {rad }}^{-1}$ with respect to the nonradiative recombination rate $\tau_{\text {nrad }}^{-1}$ (see Sec. V) as well as to optimize the light extraction efficiency $\overline{P_{e s c}}$, which will be further discussed in Sec. VI. The factors that limit the open-circuit voltage were recently elucidated by Mann et $a .^{21}$ They measured a $V_{o c}$ of $850 \mathrm{mV}$ for their single InP nanowire solar cell. In order to understand the open circuit voltage, they measured the external photoluminescence efficiency by integrating sphere microscopy. As shown in Fig. 5, the external photoluminescence efficiency was measured to be $4 \times 10^{-4}$ at an illumination intensity of 1 sun, resulting in a loss in $V_{o c}$ of $210 \mathrm{mV}$ according to Eq. (8). 


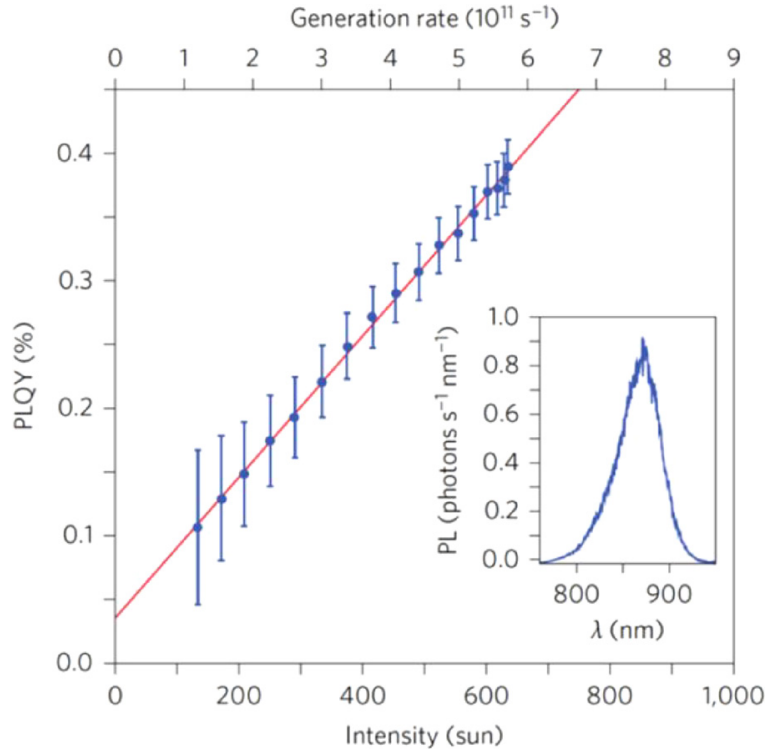

FIG. 5. Extrapolation of the measured external photoluminescence efficiency towards an illumination of 1 sun, showing $\eta_{\mathrm{ext}}^{\mathrm{PL}}=4 \times 10^{-4}$. Reproduced with permission from Mann et al., Nat. Nanotechnol. 11, 1071-1075 (2016). Copyright 2016 Springer Nature.

In order to further evaluate the performance of this single nanowire solar cell, it is necessary to compare the experimentally measured $V_{o c}$ with the radiative limit. To calculate the radiative limit for the $V_{o c}$, one needs to first calculate ${ }^{21}$ the black-body recombination current

$$
I_{\text {Black-body }}=\iint \sigma_{E Q E}(\lambda, \Omega) \varphi_{\text {black-body }}(T, \lambda) \cos \theta d \lambda d \Omega,
$$

which is an integral over the black body photon flux density $\varphi_{\text {black-body }}(T, \lambda)$ and the external quantum efficiency cross section $\sigma_{E Q E}(\lambda, \Omega)$ which was defined in Eq. (1). This integral was evaluated by Mann et al., ${ }^{21}$ yielding a black-body current of $3.1 \times 10^{-17} \mathrm{pA}$. This corresponds to a radiative limit of $V_{o c}^{r a d}=1.16 \mathrm{~V}$, which is slightly higher than the Shockley-Queisser limit. This can be explained by the reduced EQE cross section ${ }^{20,21}$ near the semiconductor bandgap. Kirchhoff's law states that the absorptivity should be equal to the emissivity. A reduced emissivity thus also reduces the solar light absorption, which makes it impossi$\mathrm{ble}^{36}$ to beat the Shockley Queisser limit. By comparing the experimental $V_{o c}$ of $850 \mathrm{mV}$ with the radiative limit, the authors find that this cell not only suffers from a $210 \mathrm{mV}$ loss due to a poor external radiative efficiency but also suffers an additional loss of approximately $100 \mathrm{mV}$, which can be attributed to the contacts. As mentioned earlier, the $V_{o c}$ is strictly speaking only related to the LED quantum efficiency; ${ }^{32}$ the relation between the $V_{o c}$ and the external quantum yield only holds in the case of perfect contacts.

\section{STATE-OF-THE-ART NANOWIRE SOLAR CELLS}

Pioneering work on nanowires and nanowire solar cell has been performed by the groups of Yang, ${ }^{37-40}$ Lieber, ${ }^{1,41,42}$ Tsakalakos, ${ }^{43,44}$ Alivisatos, ${ }^{45}$ Fukui, ${ }^{46-49}$ Jagadish, ${ }^{50-53}$ Atwater, ${ }^{54,55}$ Samuelson, ${ }^{56-59}$ Fontcuberta i Morral, ${ }^{60-63}$ Cui, ${ }^{64,65}$ and others. ${ }^{66,67}$ Much of this work has already been reviewed before ${ }^{1-9}$ and will not be treated again in the present review.

The first nanowire solar cell hitting the 10\% efficiency barrier was developed by the Lund team ${ }^{14}$ and was based on InP nanowires with an axial p-i-n junction. The nanowires had a diameter of $180 \mathrm{~nm}$ and a height of $1.5 \mu \mathrm{m}$, as shown in Fig. 6(a). This cell featured a $13.8 \%$ power conversion efficiency and a $V_{o c}$ of $0.779 \mathrm{~V}$. The paper also reported a $V_{o c}$ of $0.906 \mathrm{~V}$ for another cell with lower current (and thus lower efficiency). This result was very intriguing as it outperformed the $V_{o c}$ of the record planar InP solar cell at that time. During the same year, efficiencies of $11.1 \%$ for an axial $\mathrm{InP}$ cell $^{68}$ and $10.2 \%$ for a GaAsP cell ${ }^{69,70}$ were reported. The next record was established by a Lund University spin-off company ${ }^{15}$ by using axial p-n doped GaAs nanowires with a diameter of $165 \mathrm{~nm}$ and a pitch of $400 \mathrm{~nm}$. Since GaAs has a much higher surface recombination velocity than InP, the GaAs nanowire cores were passivated with a $25-40 \mathrm{~nm}$ thick AlGaAs radial shell. The resulting efficiency was $15.3 \%$ with a $V_{o c}$ of $0.906 \mathrm{~V}$ and a $\mathrm{J}_{\mathrm{sc}}$ of $21.3 \mathrm{~mA} / \mathrm{cm}^{2}$. Both these cells were bottom-up grown. The present world record nanowire solar cell ${ }^{16}$ was fabricated by using a top-down etched nanowire array, which is shown in Fig. 6(b) and Table I.

This cell comprises of $1.6 \mu \mathrm{m}$ tapered InP nanocones with a pitch of $513 \mathrm{~nm}$, a diameter of $150 \mathrm{~nm}$ at the top, and $350 \mathrm{~nm}$ at the base. This cell features a very efficient solar light absorption due to nanowire tapering and Indium Tin Oxide (ITO) "nanolenses" embedded within the top-contact layer (see Sec. IV). In addition, the cell features both a frontsurface field and a back-surface field to limit minority carrier recombination losses at the contacts. The resulting power
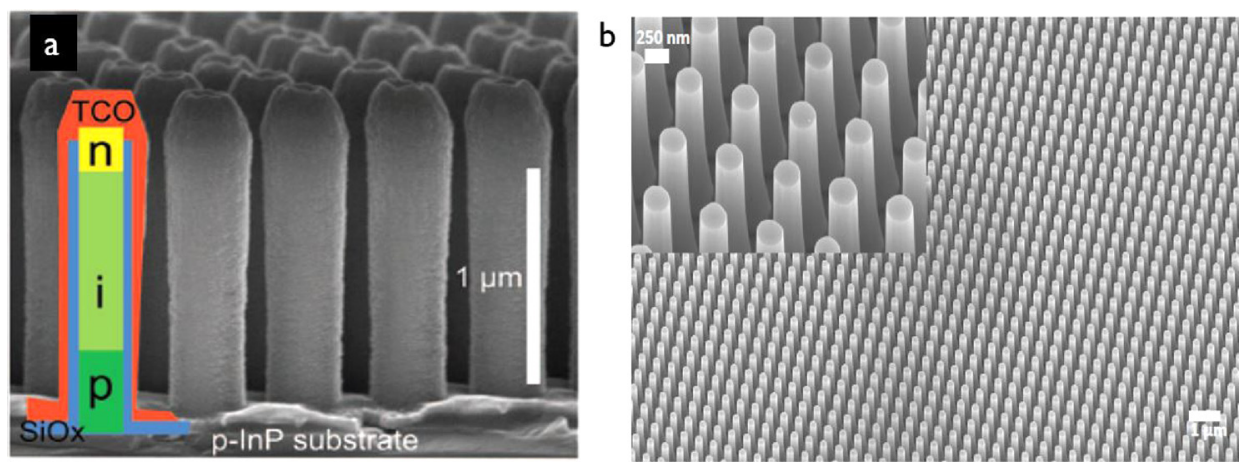

FIG. 6. Scanning electron microscopy image (a) of the processed bottom-up grown nanowires of the $13.8 \%$ efficiency ${ }^{14} \mathrm{InP}$ nanowire solar cell from Lund and (b) the tapered nanocone array (not yet processed into a solar cell) of the $17.8 \%$ InP solar cell ${ }^{16}$ from Eindhoven. Panel (a) is reprinted with permission from Wallentin et al., Science 339, 1057-1060 (2013). Copyright 2013 American Association for the Advancement of Science. 
TABLE I. Comparison between the open circuit voltage of the record $17.8 \%$ solar cell, the Shockley Queisser limit, the ultimate limit for direct solar radiation, and the InP bandgap. At the ultimate limit, the expected $\mathrm{V}_{\text {oc }}$ is slightly larger than the InP bandgap due to the excess thermal energies of the electrons and holes above the bandgap, see Eq. (16) in Sec. VIII.

\begin{tabular}{cccc}
\hline \hline InP & $17.8 \%$ efficiency cell & Shockley Queisser limit & Ultimate efficiency limit \\
\hline $\mathrm{V}_{\mathrm{oc}}=0.765 \mathrm{~V}$ & $\mathrm{~V}_{\mathrm{oc}}=1.037 \mathrm{~V}$ & $\mathrm{~V}_{\mathrm{oc}}=1.350 \mathrm{~V}$ & Bandgap \\
\hline \hline
\end{tabular}

conversion efficiency is $17.8 \%$ for the exposed area of the cell, with a $J_{s c}$ of $29.3 \mathrm{~mA} / \mathrm{cm}^{2}$ and a $V_{o c}$ of $0.765 \mathrm{~V}$.

It has been suggested that the open-circuit voltage of a nanowire solar cell can be increased due to the effect of nano-concentration. ${ }^{19,71}$ This statement is not correct for a lossless solar cell, while the statement is true for a solar cell with a low internal radiative efficiency.

The discussion on nano-concentration has been triggered by the paper of Krogstrup et al. ${ }^{19}$ They reported an apparent photo-generated current density of $180 \mathrm{~mA} / \mathrm{cm}^{2}$ for a single vertically standing GaAs nanowire solar cell. This current was obtained by normalizing the generated current with the geometrical cross section of the nanowire. The high short-circuit current density is clearly due to the fact that the absorption cross section of this nanowire was estimated to be $12 \times$ larger than its geometrical cross section, which can be explained by the nano-antenna effect (see Fig. 2). We like to emphasize that whatever the design of the nanostructure, on an array level, a nanostructured solar cell will never be capable of harvesting more current than the number of solar photons impinging on the equivalent total array surface area. The nano-antenna effect is thus only useful to limit the amount of material required to fabricate the nanostructured solar cell, which could reduce bulk recombination and potentially lower material cost.

The interesting aspect of this paper is that it suggests the possibility to enhance the $V_{o c}$ by an amount of $\frac{k T}{q} \ln (C)$ in which $C$ is the amount of nano-concentration. In a concentrator solar cell in which the solar light is concentrated by an external lens, the $\frac{k T}{q} \ln (C)$ term increases the efficiency from $33.7 \%$ towards $46.7 \%$ for direct solar radiation by increasing the concentration factor from 1 towards $46050 .^{72,73}$

For a lossless cell, Eq. (6) states

$$
\begin{aligned}
V_{\text {oc }}^{\text {rad }}\left(\frac{\varepsilon_{\text {in }}}{\varepsilon_{\text {out }}}\right) & =\frac{k_{B} T}{q} \ln \frac{\varepsilon_{\text {in }} \int a(E) \varphi_{\text {sun }}(E) d E}{\varepsilon_{\text {out }} \int a(E) \varphi_{\text {Black-body }} d E} \\
& =\frac{k_{B} T}{q} \ln \frac{R_{\text {sun }}}{R_{\text {black-body }}},
\end{aligned}
$$

which implies that the open circuit voltage is only dependent on the ratio of the solar generation rate $R_{\text {sun }}$ and the blackbody emission rate $R_{\text {black-body }}$ at short-circuit conditions. This ratio is increased for solar concentration with an external lens. An external lens is increasing the solar generation rate $R_{\text {sun }}$ but is not affecting the black-body emission rate $R_{\text {black-body }}$. Solar concentration with external optics is thus increasing the $V_{o c}$, which is a well-known result.

For nano-concentration, however, both the solar generation rate $R_{\text {sun }}$ and the black-body emission rate $R_{\text {black-body }}$ are proportional to the absorbance $a(E)$, showing that an enhanced absorbance of a nanostructure does not increase the $V_{o c}$. Mann et al. ${ }^{26}$ provide a similar argument, showing that both $R_{\text {sun }}$ and $R_{\text {black-body }}$ are proportional to the same absorption cross section. The nano-antenna effect is thus not increasing the $V_{o c}$ and nano-concentration is not creating any advantage for a lossless cell.

The story is completely different for a realistic nanowire solar cell with a small internal radiative efficiency. In this case, the active volume of the nanowire solar cell is reduced with a factor $f$ as compared to a planar cell with the same height. For an array of cylindrical nanowires with diameter $d$ and pitch $p$, the volume reduction factor becomes $\frac{p^{2}}{\pi\left(\frac{d}{2}\right)^{2}}$. The nano-antenna effect allows a single nanowire to, in principle, harvest all solar light impinging onto its absorption cross section (see Fig. 2). When the absorption cross section of neighboring nanowires is overlapping, the amount of light absorption will be the same as in a planar cell. The reduced nanowire volume, however, reduces the number of nonradiative recombination centers in the internal volume of the nanowires. Ignoring surface recombination (see Sec. VI for a justification), the nonradiative recombination loss is thus reduced with a factor $f$ relative to a planar layer, leading to an internal radiative efficiency $\eta_{\text {int }}=\frac{J_{0}^{\text {rad }}}{J_{0}^{\text {rad }}+\left(\frac{1}{f}\right) J_{0}^{\text {rrad }}} \approx \frac{f J_{0}^{\text {rad }}}{J_{0}^{\text {rada }} \text {. The }}$ IQE is thus increased with a factor $f$ due to nanoconcentration in the limit that $J_{0}^{\text {rad }} \ll J_{0}^{\text {rrad }}$.

The same results are obtained by treating a single nanowire in which the injection level is increased by a factor $f$ due to the nano-concentration effect. In this approach, the radiative emission rate increases with a factor $f$, while the nonradiative rate is determined by the number of nonradiative recombination centers and does not increases with $f$. This approach yields $\eta_{i n t}=\frac{f J_{0}^{\text {rad }}}{f J_{0}^{\text {rad }}+J_{0}^{\text {rrad }}} \approx \frac{f J_{0}^{\text {rad }}}{J_{0}^{\text {mrad }}}$ in the limit that $J_{0}^{\text {rad }} \ll J_{0}^{\text {nrad }}$, which is identical to the result obtained above. Assuming for the moment that the surface recombination can be neglected, for which we will show experimental evidence in Sec. VI, the open-circuit voltage is expected to increase ${ }^{74}$ with a term $\frac{k T}{q} \ln (f)$ as illustrated in Fig. 7. It will be shown in Sec. IV that an array of $180 \mathrm{~nm}$ diameter cylindrical nanowires with a pitch of $500 \mathrm{~nm}$ efficiently absorbs the light, leading to a nano-concentration factor $f=10$, corresponding to an increase in $60 \mathrm{mV}$ in the $V_{o c}$. For tapered nanocones, a somewhat smaller nano-concentration factor is expected due to a larger diameter at the base of the nanocone.

\section{EFFICIENT LIGHT ABSORPTION FOR A HIGH SHORT-CIRCUIT CURRENT}

Before turning towards the main focus of this review, which is on the $V_{o c}$, we first like to highlight the advantages of nanowire array solar cells for efficient solar light 


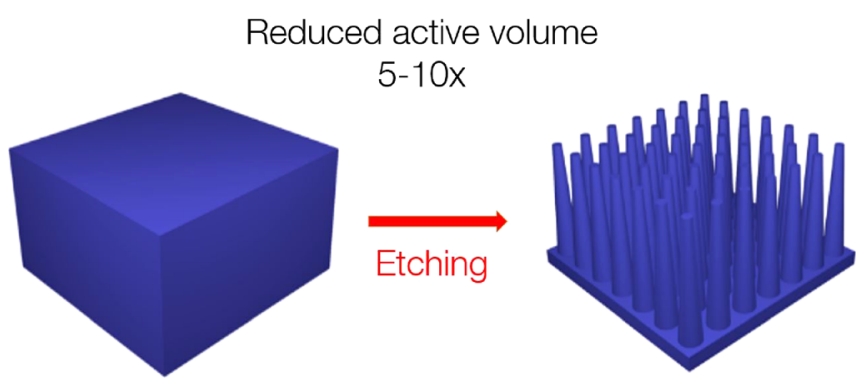

FIG. 7. The active volume of a nanowire solar cell is reduced with a factor $\mathrm{f}$, resulting in an enhanced $V_{o c}$ proportional with $\frac{\mathrm{kT}}{q} \ln (\mathrm{f})$ as $\operatorname{long}$ as $\eta_{\text {int }}^{\text {PL }} \ll 1$. The nanowire solar cell features a similar light absorption probability as a planar solar cell with identical area, provided that the absorption cross sections of the individual nanowires are overlapping. Similar light absorption with a lower amount of material features a higher $V_{o c}$.

absorption and current generation. ${ }^{75-98}$ Solar cells need to absorb light across a very broad spectral region. It is difficult to reduce the front surface reflection loss with an antireflection coating since such a coating is often only efficient in a limited wavelength region. Nanowire solar cells feature a low refractive index of the nanowire medium, resulting in a low reflection loss over a broad spectral range. By using the effective medium approach for the refractive index, ${ }^{2,65}$ the average refractive index of an array of, e.g., $100 \mathrm{~nm}$ diameter nanowires with $500 \mathrm{~nm}$ pitch and refractive index of 3.5, which are separated by air, results in an effective index of refraction of 1.14 and a reflection loss of $R$ $=\left(\frac{n-1}{n+1}\right)^{2}=0.42 \%$. This approach is over-simplified and is neglecting, e.g., diffraction effects due to the nanowire array as well as Fabry-Pérot oscillations within the nanowire layer, which are taken into account in literature. ${ }^{99}$ Although the reflection loss for a nanowire array in air can be very small, the front-surface reflection loss will substantially increase after processing these nanowires into a complete solar cell. The reason is that the nanowires will be embedded in, e.g., a polymer such as, e.g., benzocyclobutene (BCB) and that the array will be covered by, e.g., an Indium-Tin-Oxide (ITO) front contact, which increases the light reflection loss.

The strong interaction of nanowires with light due to the optical antenna effect has been reviewed by Garnett ${ }^{2}$ in 2011 and by Brongersma ${ }^{100}$ in 2014 and will not be extensively reviewed here. It suffices to say that leaky mode resonances in thin nanowires ${ }^{20,101-103}$ and the coupling of light into confined waveguide modes at sufficiently thick nanowires ${ }^{104}$ strongly enhance the optical absorption beyond the absorption expected in the ray-optics limit. ${ }^{14}$

Anttu et al. ${ }^{105}$ have performed a study to experimentally determine the optimum nanowire pitch, the optimum length, and the optimum diameter for cylindrical nanowires. In this paper, the transmission and reflection of light by a nanowire array which was embedded into a polydimethylsiloxane (PDMS) polymer were measured. Figure 8(a) shows that the absorptance for a $140 \mathrm{~nm}$ diameter nanowire saturates at 95\% for long nanowires. Although the absorptance increases with length, an axial junction nanowire solar cells with a too long nanowire length require a very long minority carrier diffusion length for achieving efficient current generation, which is very challenging. For nanowires with a length of $1.6 \mu \mathrm{m}$, one has to choose a diameter above $180 \mathrm{~nm}$ [Refs. 80, 106, and 107] [see Fig. 8(b)], above which the $\mathrm{HE}_{11}$ mode becomes a confined guided mode. At too high diameter, the reflection loss at the top of the nanowires starts to increase. Anttu et al. calculated that the reflection loss for nanowires embedded into PDMS increases from $3 \%$ to $24 \%$ when the nanowire diameter increases from 0 to $400 \mathrm{~nm}$. A larger diameter also has the disadvantage that the amount of required semiconductor material is strongly increased, which will also translate into a higher materials cost of the cell. It is found ${ }^{105}$ that $94 \%$ of the incident solar light can be absorbed by cylindrical nanowires with a length of $2 \mu \mathrm{m}$, a pitch of $400 \mathrm{~nm}$ pitch, and a diameter of $241 \mathrm{~nm}$.

For further increasing the optical absorption, it is necessary to limit the top reflectance while simultaneously increasing the light absorption. Tapered nanowires or nanocones provide a solution for this requirement since tapering establishes a graded refractive index layer which minimizes light reflection at the top facet. Consequently, tapered nanowires, in principle, allow to achieve the largest light absorption of solar light above the semiconductor bandgap. The large solar light absorption for tapered nanocones is evidenced in Fig. 9 by using Finite Difference Time Domain (FDTD) simulations. Similar results have been reported both (a)

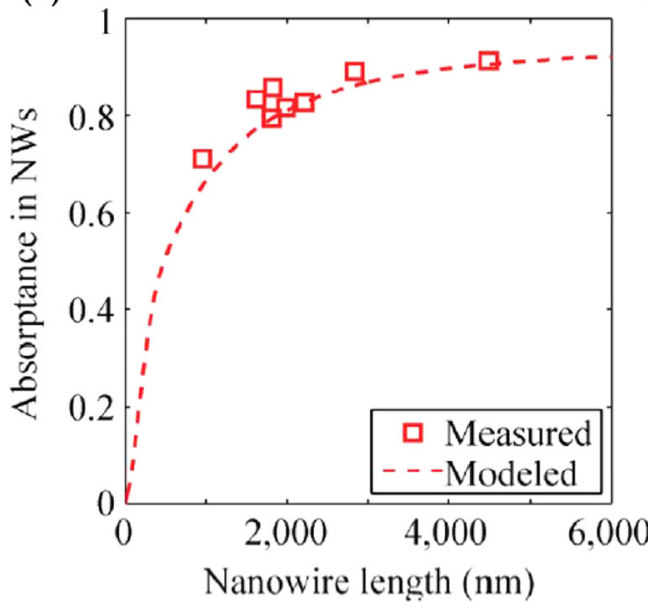

(b)

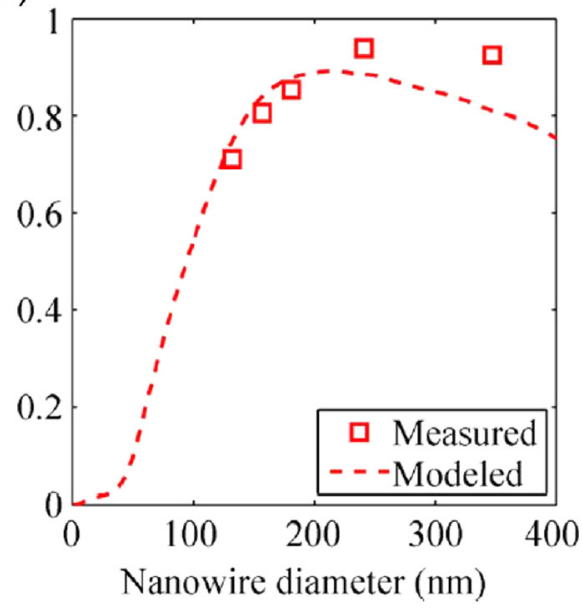

FIG. 8. Measured and simulated absorptance $^{105}$ for (a) $137 \pm 9 \mathrm{~nm}$ diameter nanowires versus length and (b) for $1600 \mathrm{~nm}$ long nanowires versus diameter. Reproduced with permission from Anttu et al., Nano Res. 7, 816-823 (2014). Copyright 2014 Springer. 


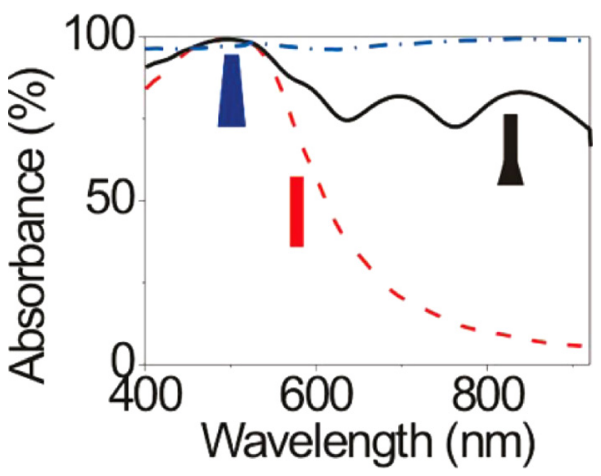

FIG. 9. FDTD simulations ${ }^{99}$ of the absorbance of $3 \mu \mathrm{m}$ length InP nanowires for normal incidence. The cylindrical nanowires (red curve) have a diameter of $90 \mathrm{~nm}$. The conical (blue curve) and base-tapered (black curve) nanowires have a diameter of $90 \mathrm{~nm}$ at the top and $270 \mathrm{~nm}$ at the bottom, where the base-tapered section has a height of $1 \mu \mathrm{m}$. The conical nanowires feature an average wavelength integrated absorption of $98 \%$. Reproduced with permission from Diedenhofen et al., ACS Nano 5, 2316-2323 (2011). Copyright 2011 American Chemical Society.

experimentally and theoretically by $\mathrm{Zhu}^{65}$ for amorphous $\mathrm{Si}$ nanocones. The reason for the enhanced absorption in the nanocones is the light coupling into several different guided modes belonging to different nanowire diameter along the nanocone, as well as by the increasing material filling fraction along the nanocone.

Although a tapered nanocone array embedded in air, in principle, allows very high optical absorption, the reflection loss in a realistic solar cell is largely determined by the reflection at the front-surface contact layer, which is typically an ITO layer. When depositing an ITO layer on top of a nanowire array in which the nanowire tips are protruding slightly above a BCB polymer layer, hemispherically shaped
ITO nanoparticles will be formed ${ }^{108}$ as shown in Fig. 10(a). These ITO hemispherical nanoparticles will forward scatter the solar light into the tapered nanocones. FDTD simulations [Fig. 10(b)] show that hemispherical Mie scatterers are efficiently scattering the light and are thus increasing the amplitude of the electric field inside the nanocones in comparison with a planar ITO contact layer. Since these Mie scatterers primarily forward scatter the solar light, these Mie scatterers also reduce the refection of the ITO contact layer. The net effect is shown in Fig. 10(c) which shows an average absorptance of $90 \%$ in the spectral region between 350 and $900 \mathrm{~nm}$ due to the forward scattering Mie particles. It is important to note that this nanowire solar cell shows only a small angle of incidence dependence [Fig. 10(d)] of the collected current up to an angle of incidence of $70^{\circ}$, which was the maximum angle accessible in the Fourier microscope setup. Similar angle of incidence dependent data has been obtained by Ghahfarokhi et al. ${ }^{109}$

We have shown that a nanocone solar cell with forward scattering ITO nanoparticles is capable to generate a shortcircuit current density ${ }^{16}$ of $29.3 \mathrm{~mA} / \mathrm{cm}^{2}$. If one takes into consideration that this current has been obtained without any detailed optimization, we conceive that a nanowire cell is able to relatively closely approach the current champion planar InP cell with a short-circuit current density ${ }^{110-112}$ of $31.15 \mathrm{~mA} / \mathrm{cm}^{2}$. In addition, our current cell is not very far from the maximum achievable short-circuit current density ${ }^{17}$ of $34.6 \mathrm{~mA} / \mathrm{cm}^{2}$ for InP. We anticipate that the short-circuit current can even be further increased by optimizing the shape of the nanocones as well as the precise shape of the forward scattering Mie scatterers. Such an optimization would not only improve the incoupling of the light and thus (a)

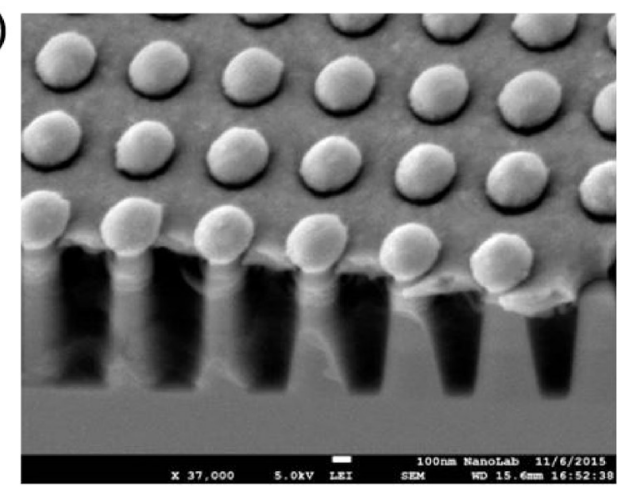

(c)

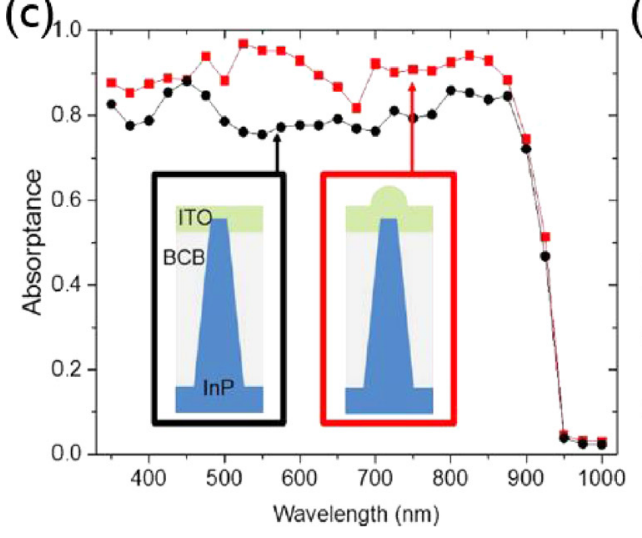

(b)

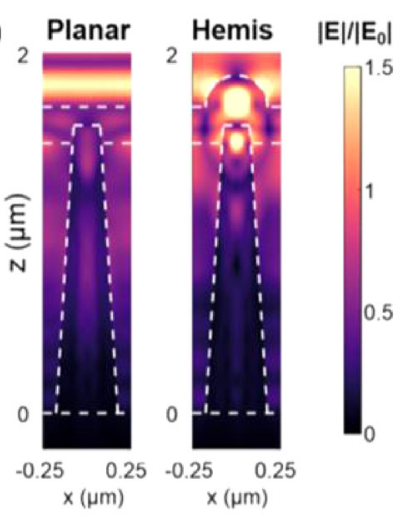

(d)

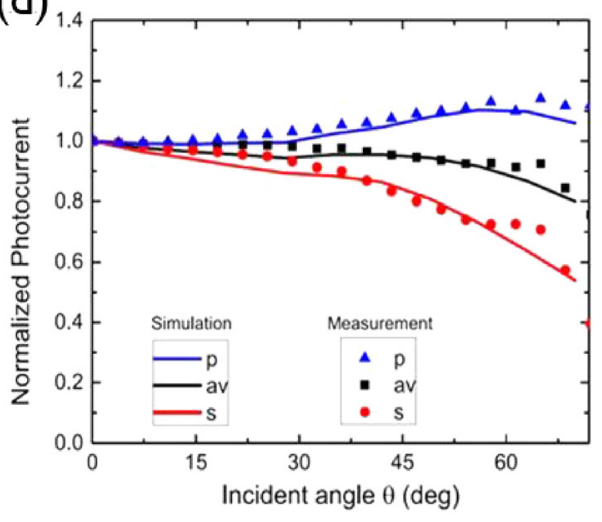

FIG. 10. (a) Scanning electron microscopy image of the cross section of the InP nanocone solar cell ${ }^{16}$ with forward Mie scattering ITO hemispheres on top of the cones. (b) FDTD simulations showing the electric field distribution inside the InP nanocones with and without the hemispherical ITO nanoparticles. (c) FDTD simulation of the absorptance of the InP nanocones with (red curve) and without (black curve) the ITO Mie scatterers. (d) Measured and simulated dependence of the photocurrent on the angle of incidence for p-polarization (blue), s-polarization (red), and the average of both polarizations (black) for incident light at $532 \mathrm{~nm}$. Reproduced with permission from Van Dam et al., ACS Nano 10, 11414-11419 (2016). Copyright 2016 American Chemical Society. 
the solar light absorption but also the light extraction probability (see Sec. VI) which is important for the $V_{o c}$.

\section{INTERNAL PHOTOLUMINESCENCE EFFICIENCY}

It is instructive to estimate the minimum required room temperature nonradiative lifetime for a GaAs nanowire solar cell for reaching an internal photoluminescence efficiency of $90 \%$ or $99 \%$. The nonradiative lifetime itself is not the relevant parameter. The key metric is the ratio between the radiative and the total (including nonradiative) recombination rate, which determines the internal photoluminescence efficiency by

$$
\eta_{\text {int }}^{P L}=\frac{\tau_{\text {rad }}^{-1}}{\tau_{\text {rad }}^{-1}+\tau_{\text {rrad }}^{-1}} .
$$

The radiative recombination rate can be expressed as $\tau_{\text {rad }}^{-1}=B n p$, where $B$ is the rate for radiative recombination. ${ }^{113}$ Using the Roosbroeck-Shockley equation ${ }^{28,114}$ which results from detailed balance requirements, the B-coefficient is calculated to be $\mathrm{be}^{23,115} B=13 \times 10^{-10} \mathrm{~cm}^{-3} \mathrm{~s}^{-1}$. It should be mentioned that considerably smaller values ${ }^{113,116}$ of initially $B=2 \times 10^{-10} \mathrm{~cm}^{-3} \mathrm{~s}^{-1}$ and more recently ${ }^{117} \quad B=3.5$ $\times 10^{-10} \mathrm{~cm}^{-3} \mathrm{~s}^{-1}$ have been reported for GaAs at low doping concentrations in carrier lifetime experiments. For InP, a Bcoefficient of $B=2 \times 10^{-11} \mathrm{~cm}^{-3} \mathrm{~s}^{-1}$ was initially reported. ${ }^{118-120}$ By taking photon recycling into account, this value was later corrected to $\mathrm{be}^{24} B=1.2 \times 10^{-10} \mathrm{~cm}^{-3} \mathrm{~s}^{-1}$ for $2 \times 10^{18} / \mathrm{cm}^{3} \mathrm{n}$-doped InP. The latter value corresponds to an internal radiative efficiency of $97 \%$, showing that InP is capable to reach very high solar energy conversion efficiency. Yablonovitch ${ }^{15}$ has shown that the B-coefficient is not a material constant, but it is dependent on the photonic density of states and local field effects. In particular, the $B$-coefficient is strongly dependent on the refractive index of the surrounding material, implying that for a GaAs epitaxial layer on a GaAs substrate, the Roosbroeck-Shockley value should be corrected by a factor $\frac{n_{e x}^{2}+1}{2 n_{i n t}^{2}} \approx \frac{1}{2}$ yielding a predicted $B \approx$ $7 \times 10^{-10} \mathrm{~cm}^{-3} \mathrm{~s}^{-1}$ and a measured ${ }^{115} \quad B=5.5$ $\times 10^{-10} \mathrm{~cm}^{-3} \mathrm{~s}^{-1}$. Yablonovitch suggests that the large scatter in reported B-coefficients is due to undefined local refractive index geometries in experiments. Unfortunately, this claim has not been experimentally verified in more recent work.

The radiative recombination rate can be expressed in terms of the $n p$ product

$$
\frac{1}{\tau_{\text {rad }}}=B(n+\Delta n)(p+\Delta p),
$$

in which $n$ and $p$ are the doping impurity concentrations and $\Delta n$ and $\Delta p$ are the solar excited carrier concentrations. In a p-doped semiconductor with acceptor concentration $N_{A}$ at low injection $\left(\mathrm{p}=\mathrm{N}_{\mathrm{A}}, \Delta \mathrm{p} \ll \mathrm{p}, \mathrm{n}=\mathrm{N}_{\mathrm{D}} \approx 0\right)$, the radiative rate is actually increased to

$$
\frac{1}{\tau_{\text {rad }}}=B N_{A} \Delta n .
$$

At a realistic doping concentration of $N_{A}=3 \times 10^{17} \mathrm{~cm}^{-3}$, the radiative emission lifetime per electron is expected to be $2.6 \mathrm{~ns}$ by assuming $B=13 \times 10^{-10} \mathrm{~cm}^{-3} \mathrm{~s}^{-1}$. The required nonradiative lifetime for reaching an internal radiative efficiency of $90 \%(99 \%)$, is thus $23 \mathrm{~ns}(253 \mathrm{~ns})$ at room temperature.

In planar III/V semiconductors, several authors measured $^{121,122}$ room temperature minority carrier lifetimes of more than $1 \mu \mathrm{s}$. In particular, Olson et al. ${ }^{123}$ achieved a 14 $\mu$ s lifetime in an $\mathrm{InGaP} / \mathrm{GaAs} / \mathrm{InGaP}$ double heterostructure grown by Metal Organic Vapour Phase Epitaxy. In addition, Ahrenkiel et al. ${ }^{16,124}$ observed a $4.84 \mu$ s lifetime in a lattice matched $\operatorname{InAs}_{\mathrm{y}} \mathrm{P}_{1-\mathrm{y}} / \mathrm{In}_{\mathrm{x}} \mathrm{Ga}_{1-\mathrm{x}} \mathrm{As}$ double heterostructure and a $21.45 \mu$ s (initial) carrier lifetime in a lattice matched InP/ $\mathrm{In}_{0.53} \mathrm{Ga}_{0.47} \mathrm{As} / \mathrm{InP}$ double heterostructure at a background doping concentration of $2 \times 10^{14} \mathrm{~cm}^{-3}$. It is well known that the coefficient for radiative recombination $B$ varies ${ }^{125,126}$ as $B=B_{0}\left(\frac{300 \mathrm{~K}}{T}\right)^{3 / 2}$, implying that pure radiative recombination lifetime in a bulk semiconductor increases with $T^{n}$ in which $n=1.5$. The reported ultralong lifetimes in planar semiconductors were found to increase with $T^{n}$ with $n \approx 1.59$, pointing towards almost pure radiative recombination. The situation for $(4-5) \times 10^{16} \mathrm{~cm}^{-3} \mathrm{n}$-doped and $\mathrm{p}$-doped $\mathrm{InP}$ is, however, different since the measured bulk lifetimes ${ }^{127}$ were found to be $320 \mathrm{~ns}$ and $4 \mathrm{~ns}$, respectively. For n-doped InP, the recombination is still radiative, ${ }^{118,119,127}$ but $p$ doped InP has a short lifetime due to electron-acceptor recombination as shown in Fig. 11. Doping related nonradiative recombination centers have also been reported for Sulphur doped InP nanowires. Zhang et al. ${ }^{128,129}$ showed that Sulphur introduces hole traps which strongly decrease the lifetime and thus also degrade the internal photoluminescence efficiency. We emphasize that more doping studies are required in the future to establish $\mathrm{p}-\mathrm{n}$ doped nanowires which are capable to achieve a high internal photoluminescence efficiency at open circuit conditions. (a)

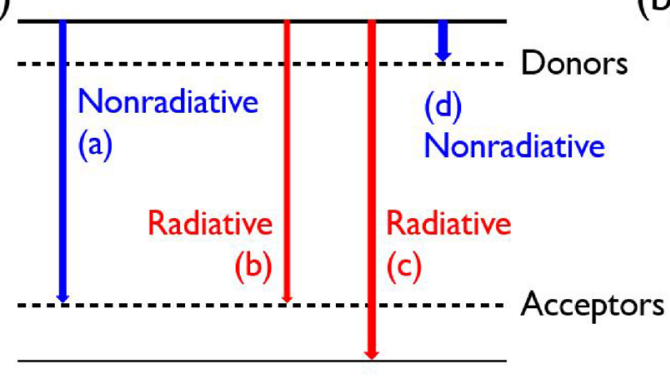

(b)

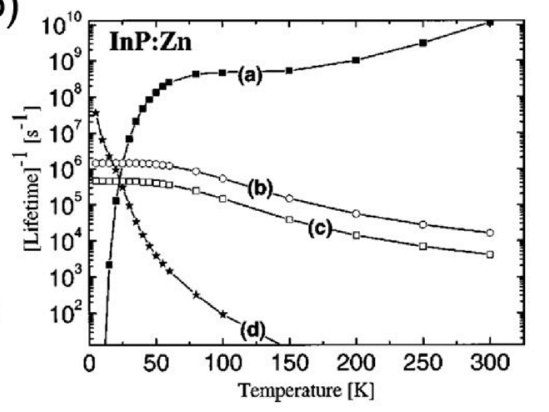

FIG. 11. Recombination rates in 4 $\times 10^{16} \mathrm{~cm}^{-3}$ p-doped planar InP, showing that the nonradiative recombination of free electrons into the $\mathrm{Zn}$-acceptor ${ }^{118}$ (a) is the dominant recombination process at room temperature. Reproduced with permission from Rosenwaks et al., Phys. Rev. B 68, 115210 (2003). Copyright 2003 American Physical Society. 
For a nanowire, the effective nonradiative recombination lifetime $\tau_{\text {eff }}$ can be expressed as ${ }^{130}$

$$
\frac{1}{\tau_{\text {eff }}}=\frac{1}{\tau_{\text {volume }}}+\frac{4 S}{d},
$$

in which $\tau_{\text {volume }}$ is a time constant for recombination at volume defects including nonradiative recombination centers due to doping, $S$ is the recombination velocity at the nanowire surface, and $d$ is the nanowire diameter. Recombination at the end facets is ignored in this equation but certainly becomes important for approaching the radiative limit. For achieving, e.g., a $23 \mathrm{~ns}$ effective recombination lifetime using Eq. (15), the surface recombination velocity should be smaller than $196 \mathrm{~cm} / \mathrm{s}$ for a $180 \mathrm{~nm}$ diameter nanowire solar cell, which is the optimum diameter for light absorption.

While the reported minority carrier lifetimes for planar double heterostructures are in the microsecond range, the reported lifetimes for III/V nanowires are usually only a few nanoseconds, or even less. There are several possible reasons for the rather low minority carrier lifetimes reported so far. A first issue is surface recombination, which is relatively low for InP, but very high for unpassivated GaAs. A second issue is nonradiative bulk recombination, possibly due to $\mathrm{Au}-$ contamination during vapor-liquid-solid (VLS) growth. Nonradiative volume recombination can also be due to point defects, ${ }^{131}$ e.g., by phosphorous out-diffusion in InP, creating phosphorous vacancies. Electron-acceptor recombination is another possible issue for p-doped InP and might impede high solar cell efficiencies for InP. In addition, during VLS growth, radial growth through the vapor-solid growth mechanism is forming an unintentional shell. Since the growth conditions are not optimized for radial growth, the unintentional shell might reduce the optical quality ${ }^{132}$ as well as the solar cell performance. ${ }^{68}$ The growth of a high bandgap shell will subsequently bury the low quality material below the shell, making such a passivation useless. Finally, any contamination during growth or accumulated on the nanowire surface after growth will immediately deteriorate the minority carrier lifetime. Although none of these problems are fundamental, we think that these issues are the reason that nanowire solar cells hitherto failed to reach high efficiencies.
Black et $a l .{ }^{133}$ recently plotted both the measured lifetime versus excitation density and the internal photoluminescence efficiency versus nanowire diameter for nominally undoped InP nanowires, as shown in Fig. 12. In these plots, these authors compare the results published by different authors ${ }^{128,130,134-140}$ with their own as-grown InP nanowires as well as with InP nanowires passivated with $\mathrm{PO}_{\mathrm{x}}$ / $\mathrm{Al}_{2} \mathrm{O}_{3}$, deposited by atomic layer deposition (ALD). Although bulk InP is not featuring the longest reported minority carrier lifetime, the $5.4 \mathrm{~ns}$ lifetime from Black et al. is still one of the best results published so far. GaAs nanowires ${ }^{141-143}$ are another option for fabricating nanowire solar cells. GaAs is featuring a much longer minority carrier lifetime in bulk, but it has a very high surface recombination velocity, which requires surface passivation. Boland et al. ${ }^{144}$ reported a $3.8 \mathrm{~ns}$ lifetime in self-catalyzed n-GaAs nanowires by engineering the band bending by selective doping. Other authors ${ }^{145-151}$ studied a higher bandgap shell for passivating the surface, resulting in a minority carrier lifetime up to $1.9 \mathrm{~ns}$. Furthmeier et al. ${ }^{152}$ reported a $11.2 \mathrm{~ns}$ exciton lifetime at $5 \mathrm{~K}$ in a wurtzite GaAs nanowire, but a room temperature lifetime was not reported. It is noteworthy to discuss the result of Breuer et al. ${ }^{153}$ They reported an increase in the minority carrier lifetime from 9 ps for $\mathrm{Au}$ assisted growth towards $2.5 \mathrm{~ns}$ by using Au-free growth, thus suggesting that an Au-catalyst nanoparticle should be avoided for the growth of GaAs nanowires for solar cell applications. This result is, however, debated since, e.g., Jiang et al. ${ }^{146}$ observed a $1.9 \mathrm{~ns}$ minority carrier lifetime in Au-catalyzed GaAs nanowires, while Aberg et al. ${ }^{15}$ observed a $15.3 \%$ solar cell conversion efficiency for Au-catalyzed GaAs nanowires, both indicating that the Au-catalyst is not severely degrading the lifetime. Recently, a nominally undoped InP/InGaAs/InP heterostructure nanowire, fabricated by top-down etching, showed a $22.8 \mathrm{~ns}$ lifetime ${ }^{154}$ for a $275 \mathrm{~nm}$ diameter pillar passivated by sulfur and protected by $\mathrm{SiO}_{\mathrm{x}}$.

The other approach is to try to increase the radiative recombination rate. Burgess et al. ${ }^{155}$ reported an external radiative efficiency of $>1 \%$ by increasing the radiative rate through doping. Tran et al. ${ }^{138}$ measured the Fermi-level splitting in $1.3 \mu \mathrm{m}$ thick micropillars which show a $>7.4 \mathrm{~ns}$ lifetime at room temperature. [The large lifetime is explained (a)

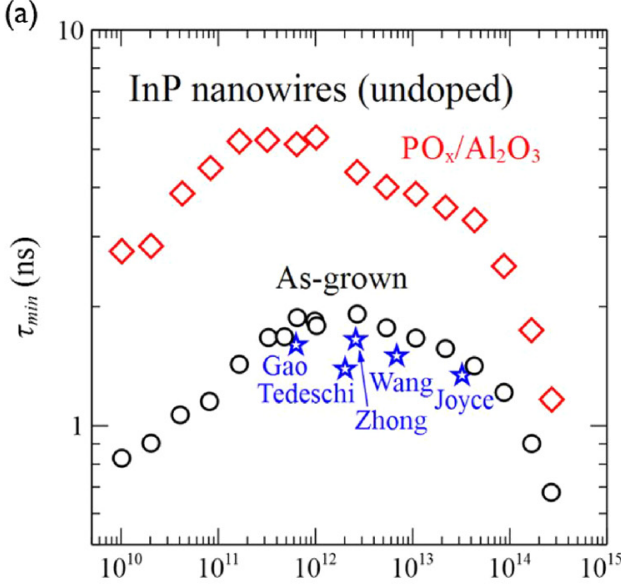

Excitation Intensity (photons $/ \mathrm{cm}^{2} /$ pulse)

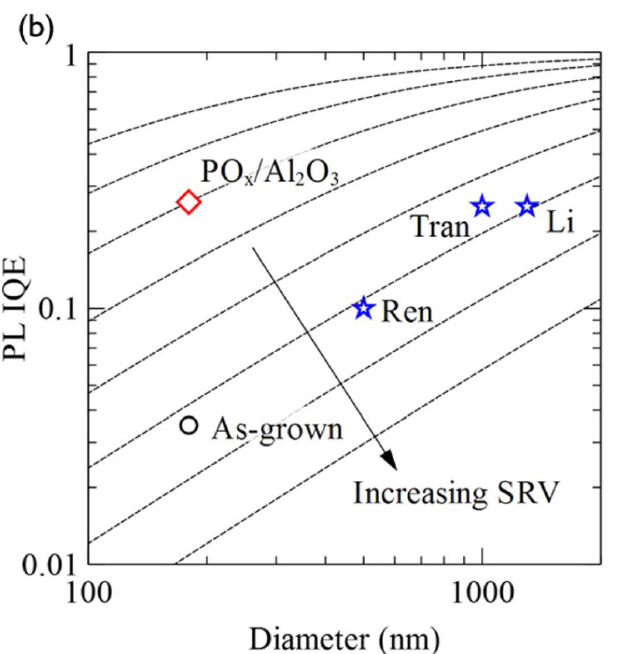

FIG. 12. (a) Comparison of measured ${ }^{133}$ carrier lifetimes versus excitation intensity for both passivated and as-grown InP nanowires, compared with the literature studies. ${ }^{128,130,134,135,137}$ (b) Internal photoluminescence efficiency (IQE) at $300 \mathrm{~K}$ for undoped InP nanowires versus diameter, compared with the literature studies. ${ }^{136,138,139}$ The contour lines show the expected IQE versus diameter for surface related recombination and constant surface recombination velocity. Reproduced with permission from Black et al., Nano Lett. 17, 6287-6294 (2017). Copyright 2017 American Chemical Society. 
(a)

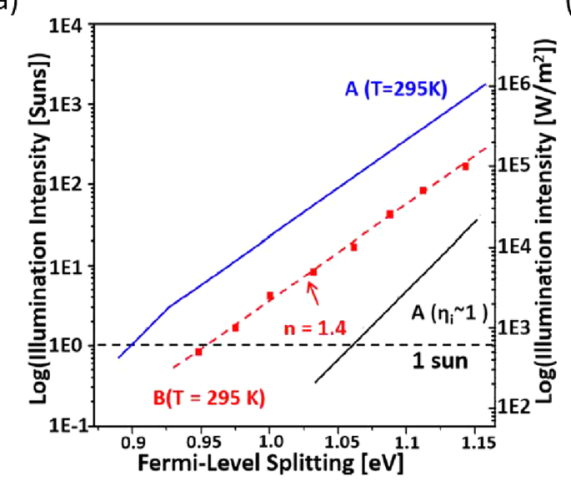

(b)

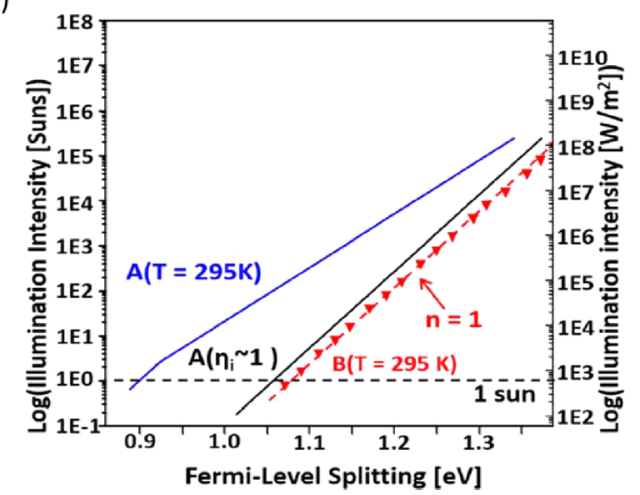

FIG. 13. Measured ${ }^{138}$ quasi Fermi-level splitting versus illumination intensity at 295 K. Panel (a) shows a comparison between untreated nanopillars (blue curve) and piranha etched "cleaned" nanopillars (red curve) with a Fermi-level splitting of $0.95 \mathrm{~V}$ at 1 sun. The black curve is measured at $4 \mathrm{~K}$ and shows the ideal radiative behavior. Panel (b) shows a $4 \times 10^{18} \mathrm{~cm}^{-3} \mathrm{n}$-doped micropillar in red with an unprecedented $1.07 \mathrm{~V}$ Fermi-level splitting, in comparison with the same blue and black curves as in (a). Reproduced with permission from Tran et al., Nano Lett. 14, 3235-3240 (2014). Copyright 2014 American Chemical Society.

by Eq. (15).] These micropillars feature a $0.95 \mathrm{~V}$ quasi Fermi-splitting after surface "cleaning" by piranha etching. By increasing the n-type doping level to $4 \times 10^{18} \mathrm{~cm}^{-3}$, the radiative recombination rate was further enhanced, resulting in a quasi-Fermi-level splitting as large as $1.07 \mathrm{~V}$, as is shown in Fig. 13

In this review, we will not focus on recombination losses at the front and back contact. We, however, like to mention that for pushing the limits, recombination at the contacts and the end facets of the nanowire should be reduced by, e.g., using front surface fields and back surface fields. ${ }^{16}$ Alternatively, an approach using passivating contacts $^{156-158,204}$ might be explored to reduce recombination losses near the contacts. We think that it is fair to conclude that the first priority to approach the Shockley Queisser limit is to increase the minority carrier lifetimes at room temperature aiming at a much higher internal photoluminescence efficiency than presently achievable. Doping optimization for increasing the radiative recombination rate is another option, provided that the doping atoms are not generating additional nonradiative recombination centers.

\section{ENHANCED OPEN-CIRCUIT VOLTAGE BY AN ENHANCED PHOTON EXTRACTION PROBABILITY}

In a lossless cell, any absorbed solar photon will eventually be re-emitted. In the lossless case, it not relevant how many times the photon needs to be recycled before it is externally re-emitted. We here disregard the situation of an extremely low nonradiative recombination loss and a restricted emission angle, where photon recycling is advantageous since it can re-direct photons into the allowed emission cone. ${ }^{159}$ For a realistic solar cell with sizeable nonradiative recombination loss, it is required that the photon extraction probability is as large as possible. In this case, each photon recycling sequence introduces a probability for a loss of the photon in a nonradiative recombination event, as schematically indicated in Fig. 14.

Using the equation $\eta_{\text {ext }}^{P L} \approx \eta_{\text {int }}^{P L} \overline{P_{\text {esc }}}$, it is directly obvious that for obtaining a high $V_{o c}$, it is equally important to optimize the photon extraction probability $\overline{P_{e s c}}$, as to optimize the internal radiative efficiency $\eta_{i n t}^{P L}$. Light extraction is fundamentally different in a nanowire solar cell as compared to a planar cell. In a planar cell without an anti-reflection coating, most of the emitted photoluminescence is emitted into a random direction. A large fraction of the emitted light is subsequently totally internally reflected, resulting in an angle averaged photon escape probability ${ }^{34}$ proportional to the solid angle of the escape cone, which is $\frac{1}{4 n^{2}} \approx 2 \%$ for planar $\mathrm{InP}$, in which $n$ is the index of refraction. The low photon escape probability in a planar cell is schematically shown in Fig. 15(a). To the best of our knowledge, light extraction from a nanowire solar cell has not been experimentally investigated. The light extraction from a nanowire single photon emitter has, however, been studied, both experimentally and theoretically. In this case, the emission of a quantum dot embedded into a nanowire allows to couple $95 \%$ of the emitted light into the fundamental $\mathrm{HE}_{11}$ mode of the nanowire cavity for a nanowire diameter $d=0.22 \lambda$. In nanophotonics, the coupling probability ${ }^{160}$ into the fundamental optical mode is referred to as the spontaneous emission factor $\beta$. For off-axis dipoles, the Mie modes will also be excited, leading to a lower spontaneous emission factor for a
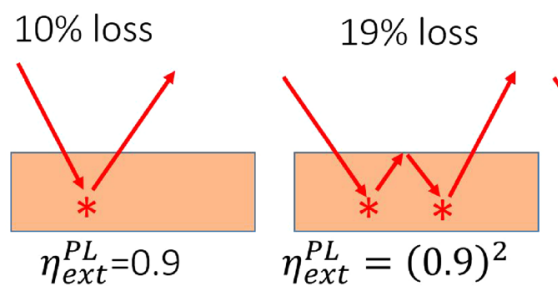

\section{$27 \%$ loss}

FIG. 14. Schematic of the photon recycling process ${ }^{22}$ in a cell with a $\eta_{\text {int }}^{\mathrm{PL}}=0.9$. The initial absorption process $(*)$ is of course required for reaching a high short-circuit current. The optimum situation is depicted in the leftmost panel in which the emitted photon leaves the cell as soon as possible, preventing a second re-absorption event. When the emitted photon is not directly coupled out, the photon has to undergo a second or even a third reabsorption-re-emission sequence, which is adding a $10 \%$ loss (for $\eta_{\text {int }}^{\mathrm{PL}}=0.9$ ) each time. Total internal reflection is thus decreasing the external radiative emission from $\eta_{\mathrm{ext}}^{\mathrm{PL}}=90 \%$ (no reflection) to $\eta_{\mathrm{ext}}^{\mathrm{PL}}=81 \%$ and $\eta_{\mathrm{ext}}^{\mathrm{PL}}=73 \%$, with one or two internal reflections. This clearly shows that the angle-averaged photon extraction probability should be as large as possible for optimizing the open-circuit voltage. 

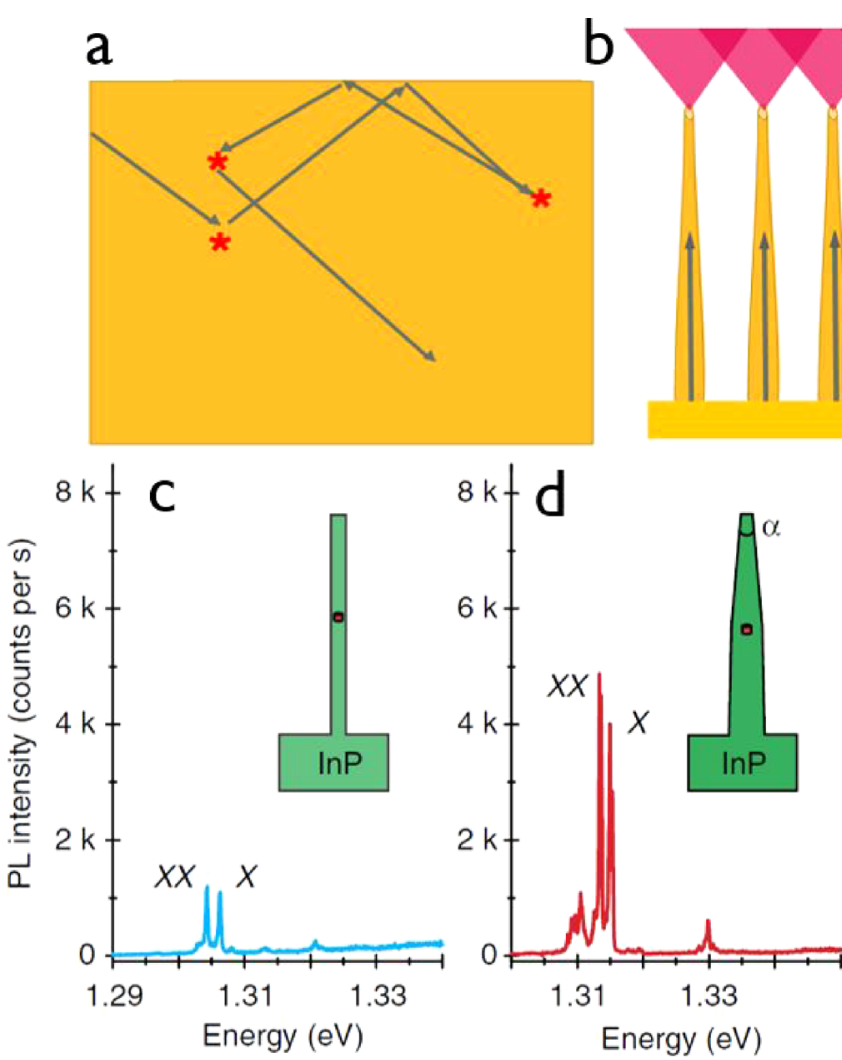

nanowire solar cell as in the case of an on-axis quantum dot emitter.

Assuming that a sizeable fraction of the emitted light in a nanowire solar cell is emitted into the $\mathrm{HE}_{11}$ mode of the nanowire, it is possible to adiabatically expand the $\mathrm{HE}_{11}$ mode into the surrounding air by gradually tapering the nanowire tip as shown in Fig. 15(b). Friedler et al. ${ }^{160}$ theoretically calculated a modal transmission of $99 \%$ with a $0.6 \%$ back radiation loss and $0.13 \%$ specular reflection for a tapered top section of the nanowire with $1 \mu \mathrm{m}$ length. Experimentally, Reimer et al. ${ }^{161}$ measured the single photon collection efficiency from a single quantum dot embedded inside a nanowire waveguide [Figs. 15(c)-15(e)]. They estimate a $42 \%$ collection efficiency for a single photon, which was measured with a 0.75 numerical aperture (NA) objective. The key for achieving such a high collection efficiency was to use a tapered nanowire with a gold back-reflector as shown in the inset of Fig. 15(e). As compared to the cylindrical nanowire shown in Fig. 15(c), the amount of collected photoluminescence was increased with a factor of 20 by using a tapered nanowire with a gold back-reflector.

Another way of increasing the external photoluminescence of a nanowire with low $\eta_{\text {ext }}^{P L}$ is by using the Purcell effect, as has recently been demonstrated by Schmitt et al. ${ }^{162}$ By tailoring the local density of optical states around the emitter, the spontaneous emission rate $\tau_{r}^{-1}$ can be enhanced by the Purcell effect into an enhanced emission rate $F / \tau_{r}$, in which $F \propto \frac{Q}{V}$ is the Purcell factor, $Q$ is the cavity quality factor, and $V$ is the cavity mode volume. In a solar cell, the Purcell effect can be very useful to enhance the radiative rate relative to the nonradiative loss. Schmitt et al. ${ }^{162}$ demonstrated whispering gallery modes in an inverted nanocone,
FIG. 15. (a) Total internal reflection at the semiconductor-air interface is limiting the light extraction of a planar cell. (b) Efficient outcoupling of light with a very low amount of backreflected radiation due to adiabatic expansion of the guided mode within the nanowire into the surrounding air. A tapered nanowire [panel (d)] with a quantum dot emitter, which is waveguiding a confined mode, features a higher external quantum dot photoluminescence ${ }^{161}$ than a non-waveguiding nanowire shown in (c). Panel (e) shows a $2.4 \times$ enhancement of the collected photoluminescence as compared to panel (d) and a $20 \times$ enhancement with respect to the non-waveguiding nanowire in (c), measured with a NA $=0.75$ objective. In the case of a quantum dot emitter, interference between forward emitted PL and back-reflected PL should be taken into account. In a solar cell, these interferences are absent due to the random position of the emitter. Panels (c)-(e) are reproduced with permission from Reimer et al., Nat. Commun. 3, 737 (2012). Copyright 2012 Springer Nature. with a Q-factor of 1300 , and observed a $200 \times$ enhancement of the external photoluminescence by this nanophotonic engineering approach.

Now that it is clear that the open-circuit voltage is critically determined by the external radiative efficiency $\eta_{\text {ext }}^{P L}$ $\approx \eta_{\text {int }}^{P L} \overline{P_{e s c}}$, which is, in turn, determined by the product of the internal radiative efficiency and the photon escape probability; ${ }^{163}$ it would be interesting to experimentally verify that a solar cell with an enhanced photon extraction efficiency also shows an increased $V_{o c}$. Unfortunately, such an experiment has not yet been performed for a completely processed solar cell.

It is, however, possible to experimentally measure the effect of the photon extraction probability on the $V_{o c}$ by a noncontact method. It has been demonstrated by Tran et al. ${ }^{138}$ that the quasi Fermi level splitting, and thus the maximum achievable $V_{o c}$ within a nanopillar shaped semiconductor, can be obtained by calibrated photoluminescence measurements. These authors proposed a plot of $\ln \left(I_{P L}\right)$ versus $\ln \left(I_{\text {excitation }}\right)$ using the equation $\ln \left(I_{P L}\right)=n \ln \left(I_{\text {excitation }}\right)+C$. In this equation, $n$ is an ideality factor which is unity for pure radiative recombination and which yields $n=2$ for nonradiative recombination. Schmidt et al. ${ }^{164}$ reported $1<n<2$ for free- and bound-exciton emission at $1.6 \mathrm{~K}$, while these authors reported $n<1$ for free-to-bound and donor-acceptor pair recombination. The latter recombination mechanisms rely on the electron concentration $n$ or the hole concentration $p$ and not on the product $n p$ which is proportional to the excitation intensity. Tran et al. ${ }^{138}$ observed the true radiative $n=1$ behavior for their InP nanopillars at $4 \mathrm{~K}$, as was already shown in Sec. V. Such a plot allows to directly compare the measured PL-efficiency with the radiative limit $I_{P L}=I_{\text {excitation }}$ with $n=1$ in the same plot. This 
procedure was also followed by Cui et al..$^{34}$ Their figure is shown here as Fig. 16, in which the radiative limit is plotted as a green line and the 1 sun condition is plotted as a vertical blue line. This plot allows to directly compare the quality of any semiconductor material with the radiative limit. The important message is that a nanowire array, which was defined by etching using the same wafer as for the planar layer, increases the external photoluminescence efficiency by a factor of 14 as compared to the planar layer. The enhanced $\eta_{\text {ext }}^{P L}$ corresponds to an enhancement of the $V_{o c}$ of $70 \mathrm{mV}$, which was calculated by using Eq. (8). A detailed analysis ${ }^{34}$ shows that $42 \mathrm{mV}$ of this enhancement is due to a lower amount of material and thus a lower number of nonradiative recombination centers, as shown schematically in Fig. 7. In addition, the light extraction efficiency was calculated to increase from $2 \%$ for a planar layer to $13 \%$ for a nanowire in which the guided optical mode adiabatically expands into air. The more efficient light extraction results in an enhancement of the $V_{o c}$ of $47 \mathrm{mV}$ due to more efficient light extraction in the nanowire array. The increased surface area finally reduces the $V_{o c}$ by $19 \mathrm{mV}$, resulting in a net enhancement of the $V_{o c}$ of $70 \mathrm{mV}$. This constitutes an experimental proof that a nanowire array can increase the open circuit voltage by more efficient light extraction.

\section{ENHANCEMENT OF THE VOC BY A REDUCED EMISSION INTO THE SUBSTRATE}

It is well known that epitaxially grown layers have a superior optical quality in comparison with the high-loss semiconductor substrate in which all spontaneous emission will quickly be completely lost. The importance of radiative emission loss into the lossy substrate has first been recognized in the paper of Miller et al. ${ }^{22}$ It was shown in this paper that a planar cell with a "bad mirror," i.e., a

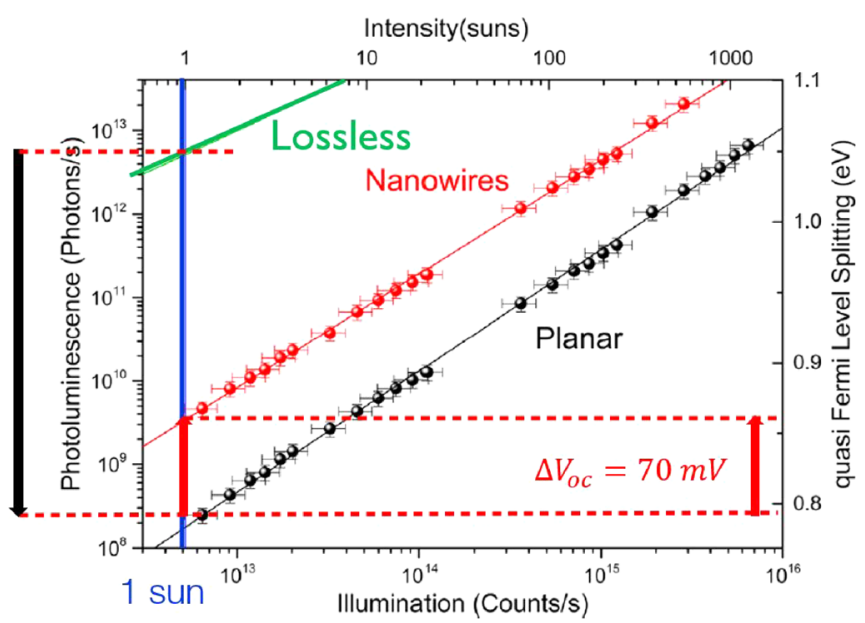

FIG. 16. Plot to determine the quasi Fermi level splitting as a function of the illumination intensity, constructed by measuring the external photoluminescence intensity. The radiative limit is plotted in green and the 1 sun condition is plotted as a vertical line in blue. The black line shows the performance of undoped planar InP with $\eta_{\mathrm{ext}}^{\mathrm{PL}}=5.2 \times 10^{-5}$ (black downward arrow), while the red line shows the performance of nanowires etched from the same source material with $\eta_{\text {ext }}^{\mathrm{PL}}=7 \times 10^{-4}$ at 1 sun. We observe a $14 \times$ enhancement of $\eta_{\mathrm{ext}}^{\mathrm{PL}}$, which corresponds to an enhancement of the open circuit voltage of $\Delta \mathrm{V}_{\mathrm{oc}}=70 \mathrm{mV}$ for the etched nanowires. Adapted with permission from Cui et al., Nano Lett. 16, 6467-6471 (2016). Copyright 2016 American Chemical Society. conventional semiconductor substrate, features a maximum $V_{o c}$ of $1.08 \mathrm{~V}$ for a $1 \mu \mathrm{m}$ thickness GaAs solar cell. When the substrate is, however, replaced by a "good mirror," the maximum $V_{o c}$ theoretically increases up to $1.15 \mathrm{~V}$. In this paper, the idea was to remove the "bad" GaAs substrate by epitaxial lift-off and to evaporate a "good" gold back-mirror at the bottom of the cell, ${ }^{165,166}$ which should also act as an electrical contact to the backside of the cell. The paper of Miller et al. ${ }^{22}$ made it very clear that the Shockley-Queisser limit can never be reached by a planar GaAs solar cell on top of a conventional high-loss GaAs substrate. On a GaAs substrate, the maximum efficiency is limited to $30.9 \%$, even for a $10 \mu \mathrm{m}$ thick cell. According to this paper, the efficiency of a GaAs cell can theoretically be improved to $33.3 \%$ by adding a high reflectivity back-mirror.

In a planar solar cell, the spontaneous emission loss into the $\mathrm{n}=3.5$ substrate is enhanced $\mathrm{d}^{22,74}$ by a factor $n^{2}$ $=(3.5)^{2}=12.25$ with respect to the emission towards the topside of the cell into the air, as shown in Fig. 17(a). The higher emission into the substrate can be explained by the enhanced optical density of states which scales with $n^{2}$. For a planar cell, the half angle of the emission cone into the substrate amounts to $90^{\circ}$. Fortunately, the emission cone for downward emission from the nanowire into the $n=3.5$ substrate is strongly limited by refraction of the light. Anttu ${ }^{74}$ calculates that the refractive index of the $\mathrm{HE}_{11}$ mode equals to $n_{H E_{11}}=1.2$, resulting in an emission cone with a halfangle of $\sin ^{-1}\left(\frac{1.2}{3.5}\right)^{2}=20.1^{\circ}$ into the $n=3.5$ substrate, as shown in Fig. 17(b). In a nanowire solar cell, the effect of the higher spontaneous emission loss into the substrate is thus largely compensated by the reduced emission cone into the substrate. A nanowire solar cell thus features a substantially lower saturation current density loss into the substrate, $e F_{c 0 \text {, bot }}$, as compared to a planar solar cell. For a solar cell without any nonradiative recombination loss, i.e., $\eta_{e x t}^{P L}=1$, the open-circuit voltage can be expressed as $V_{o c}=\frac{k_{B} T}{q} \ln \frac{J_{S c}}{J_{0}}$ $=\frac{k_{B} T}{q} \ln \frac{J_{s c}}{e F_{c 0}}$ with $F_{c 0}$ being the black-body emission of the solar cell at short-circuit conditions. The reduced black-body emission into the lossy substrate reduces the saturation current density $J_{0}$, which increases the open-circuit voltage with $100 \mathrm{mV}$ compared to a planar cell, as shown in Fig. 17(c). This results in a nanowire solar cell efficiency exceeding the efficiency of the best planar cell on a lossy semiconductor substrate with $1.5 \%$ in absolute numbers. We like to remark that it is not possible to exceed the Shockley-Queisser limit in this way, since the directivity of the emitted light has not been improved.

The open circuit voltage of a nanowire solar cell can be even further enhanced by placing a highly reflective metallic back-mirror to the bottom of the cell. In a lossless cell, such a back-mirror is increasing the directivity with a factor of 2 . The photon entropy term $\left|\frac{k_{B} T}{q} \ln \frac{\varepsilon_{i n}}{\varepsilon_{\text {ou }}}\right|$ provides a voltage increase of $\frac{k_{B} T}{q} \ln 2=18 \mathrm{mV}$ by increasing the directivity of the emitted light by a factor of 2 . For a realistic cell, this requires that the back-reflected photoluminescence is not reabsorbed, which is possible since the absorbance is small for 
(a)

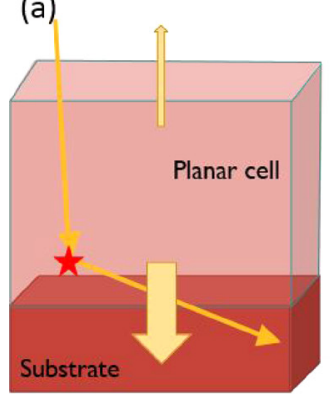

(b)

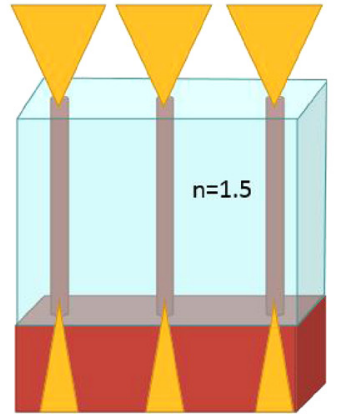

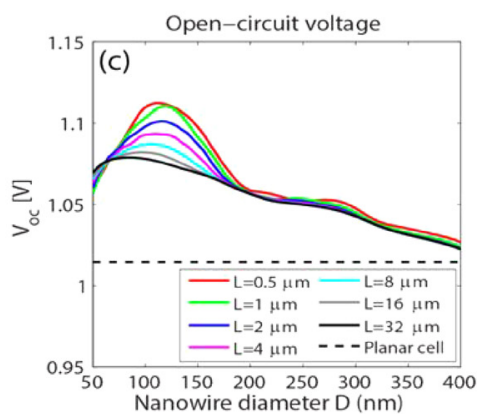

FIG. 17. A nanowire array solar cell is predicted to show a higher $\mathrm{V}_{\mathrm{oc}}$ than a planar cell. (a) The amount of black-body radiation lost into the substrate is $12.25 \times$ higher than the front side emission. (b) In a nanowire solar cell, the higher optical density of states in the substrate is counteracted by a smaller emission cone of the radiation which is lost into the substrate. The net effect is that the emission into the lossy substrate is strongly reduced and becomes almost equal to the front-side emission, which is fundamentally different from bulk. (c) The open-circuit voltage in a nanowire solar cell is up to $100 \mathrm{mV}$ higher ${ }^{74}$ as compared to a planar cell. Panel (c) is reproduced with permission from N. Anttu, ACS Photonics 2, 446-453 (2015). Copyright 2015 American Chemical Society.

photoluminescence emitted close to the bandgap. Alternatively, when the back-reflected emission is re-absorbed, it should be re-emitted with a probability of unity to obtain the $18 \mathrm{mV}$ voltage gain. A back mirror is thus reducing the photon entropy loss associated with the absorption of sunlight followed by the emission of photoluminescence. Employing a back-mirror, in principle, allows to slightly beat the Shockley Queisser limit. In practice, however, the voltage gain is too small to compensate for other almost inevitable losses, making it highly improbable that this design will ever beat the S-Q limit.

When the internal radiative efficiency is not yet unity, part of the back-reflected photoluminescence is lost. A recent paper $^{167}$ reported an increase in $60 \%$ of the front-side photoluminescence by positioning a back-mirror below an array of InP nanowires embedded into Polydimethylsiloxane (PDMS), as is shown in Fig. 18. If processed into a solar cell, a back-mirror increases the open-circuit voltage of our present nanowire solar cell by $12 \mathrm{mV}$.

\section{TOWARDS THE ULTIMATE EFFICIENCY SOLAR CELL}

Once a solar cell reaches the Shockley Queisser limit, it absorbs all projected solar radiation generating a high photocurrent. At open circuit, it is subsequently re-emitting all radiation with a $4 \pi$ solid angle. As a consequence, the solar cell is converting a nearly parallel beam of photons with solid angle ${ }^{10} \varepsilon_{\text {sun }}=6.85 \times 10^{-5} \mathrm{sr}$ into an randomized (approximately isotropic) emission pattern. The solar cell is thus working as an engine which is increasing the entropy of the solar photons. ${ }^{168,169}$ This is schematically illustrated in Fig. 19.

At the Shockley Queisser limit, the term $\frac{k_{B} T}{q} \ln \frac{\varepsilon_{\text {in }}}{\varepsilon_{\text {out }}}[\mathrm{Eq}$. (8)] reduces the $V_{o c}$ with $\sim 0.3 \mathrm{~V}$ due to the increase in photon entropy. A more precise discussion of this term can be found elsewhere. ${ }^{18,72,170-172}$ In theory, the photon entropy loss can be circumvented by sending all emitted photons back to the sun, as schematically depicted in Fig. 19. In the ultimate limit, the only remaining loss factor that reduces the $V_{o c}$ below the semiconductor bandgap is the Carnot loss. On the other hand, the $V_{o c}$ is slightly increased by a slight increase in the "apparent semiconductor bandgap" due to the average thermal energy of the charge carriers. These two effects result in ${ }^{18}$

$$
V_{\text {oc }}^{\text {Ultimate }}=\frac{E_{\text {gap }}}{e}\left(1-\frac{T_{\text {cell }}}{T_{\text {sun }}}\right)+\frac{k T_{\text {cell }}}{e} \ln \left(\frac{T_{\text {sun }}}{T_{\text {cell }}}\right),
$$

which yield an ultimate $V_{o c}$ of $10.5 \mathrm{mV}$ above the semiconductor bandgap for InP $\left(E_{\text {gap }}=1.34 \mathrm{eV}\right)$ at $300 \mathrm{~K}$.

The ultimate limit ${ }^{10}$ was first discussed in the seminal paper by Shockley and Queisser in 1961. For simplicity, they assumed a spherical sun $\varepsilon_{\text {sun }}=4 \pi=\varepsilon_{\text {out }}$ which was illuminating the (a)

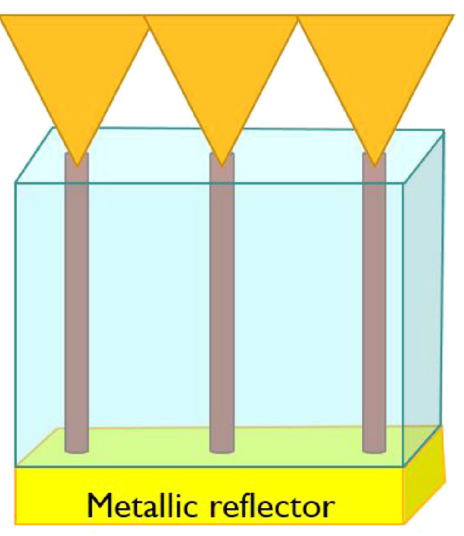

(b)

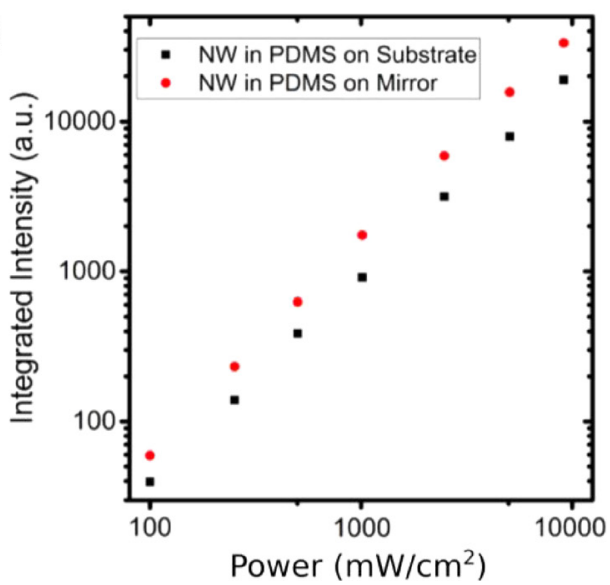

FIG. 18. (a) The open-circuit voltage of a nanowire solar cell can be increased by positioning a back-mirror at the bottom of the cell. The back-mirror increases the front-side emission. (b) Experimentally, a $60 \%$ enhancement of the front-side emission was reported by depositing a 95\% reflectivity Ag mirror below a nanowire sample embedded into PDMS. Panel (b) is reproduced from Cavalli et al., Nano-Struct. Nano-Objects 16, 59-62 (2018). Copyright 2018 Elsevier. 
(a)
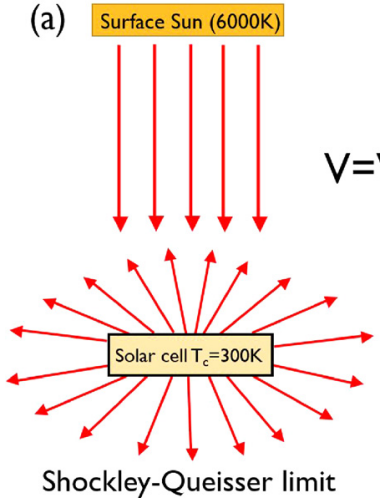

(b)

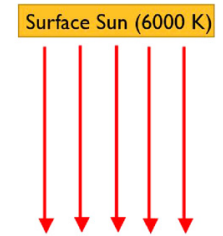

FIG. 19. (a) At the Shockley Queisser limit, a solar cell operating at opencircuit conditions is converting a parallel beam of photons originating from the sun into a spherical beam of emitted photons. This mechanism is strongly increasing the photon entropy and is responsible for a reduction of the $V_{o c}$ by $\sim 0.3 \mathrm{~V}$. (b) When a solar cell is designed to re-emit all photons back to the sun, the photon entropy is not increased and the ultimate limit is reached.

solar cell from all directions, thus avoiding any increase in photon entropy in the light absorption-emission cycle. Using the spherical sun, they already predicted an ultimate efficiency of $44 \%$ using the black-body approximation for the solar spectrum. The photon entropy effect is also wellknown in concentrator photovoltaics, where the input solid angle $\varepsilon_{i n}$ is increased by using an external concentrating lens, as shown in Fig. 20. It can be seen ${ }^{72,73}$ that the solar cell efficiency increases up to a concentration factor of $X \approx 46050$, where the ultimate limit is reached (assuming only direct solar radiation).

It has been theoretically suggested that one can exceed the Shockley Queisser limit by restricting ${ }^{18,71,173-175}$ the angle of the emission of the emitted light. A practical implementation of this idea has been investigated, ${ }^{169,176,177}$ showing that it is theoretically possible to reach efficiencies above $38 \%$ for

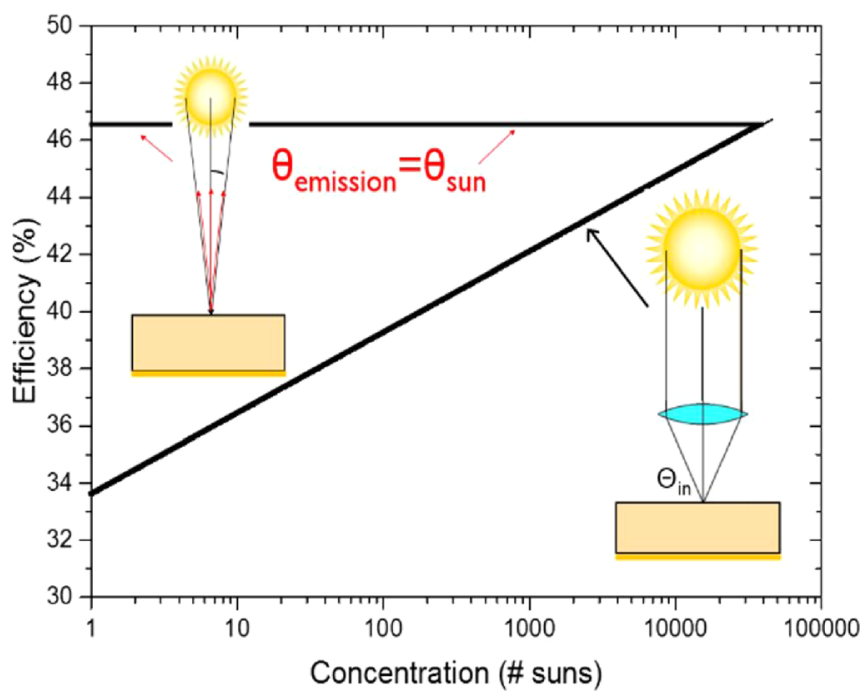

FIG. 20. Solar cell efficiency for $\eta_{\mathrm{ext}}^{\mathrm{PL}}=1$, as a function of the solar concentration by using an external focusing lens with concentration factor $X$ defined by $\sin ^{2}\left(\theta_{\text {in }}\right)=X \sin ^{2}\left(\theta_{\text {sun }}\right)$. For direct solar radiation, the ultimate limit can be either reached with a concentration factor of $46050 x$. Alternatively, the ultimate limit can be reached by focusing all emission back to the sun, yielding the horizontal line with $\theta_{\text {emission }}=\theta_{\text {sun }}$. a GaAs cell. ${ }^{169}$ Experimentally, a small voltage gain of $\sim 3.6 \mathrm{mV}$ has been observed by using angle restriction. ${ }^{177}$ Nanophotonic engineering provides new opportunities for approaching the ultimate efficiency since the light emitted at $V=V_{o c}$ is at least partially emitted into the confined optical mode of the nanowire optical cavity. We predict that this light can, in principle, be focused back towards the sun, as is schematically shown in Fig. 21. The amount of light which is emitted into the fundamental cavity mode is determined by the spontaneous emission factor of the particular nanophotonic cavity. Friedler et al. ${ }^{160}$ have calculated that for a quantum dot embedded inside a nanowire with a diameter $d=0.22 \lambda$, the spontaneous emission factor $\beta$ can be larger than $95 \%$. It should be emphasized that this number has been calculated for a quantum dot, which corresponds to an on-axis dipole. We, however, like to emphasize that even when a smaller fraction of the emitted light is coupled into the fundamental cavity mode, that light can, in principle, be focused back towards the sun, thus reducing the increase in photon entropy at open-circuit conditions. We thus predict that nanophotonic engineering allows to approach the ultimate limit.

It is important to mention that a nanophotonic ultimate limit solar cell should not increase the photon entropy anywhere along its light path. Random surface texturing is, e.g., completely detrimental for an ultimate limit cell, since a texture is increasing the entropy of the light in an irreversible

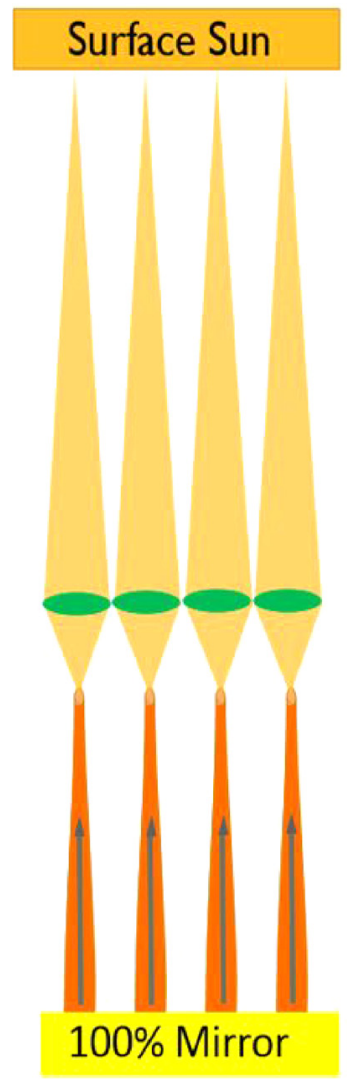

FIG. 21. Proposal for an ultimate efficiency nanowire solar cell in which all light is emitted into the fundamental guided mode of the nanowire optical cavity. This guided mode can be focused back to the sun by an external lens. It is important that all light emitted towards the bottom of the nanowires is also reflected back towards the sun at open-circuit conditions. 


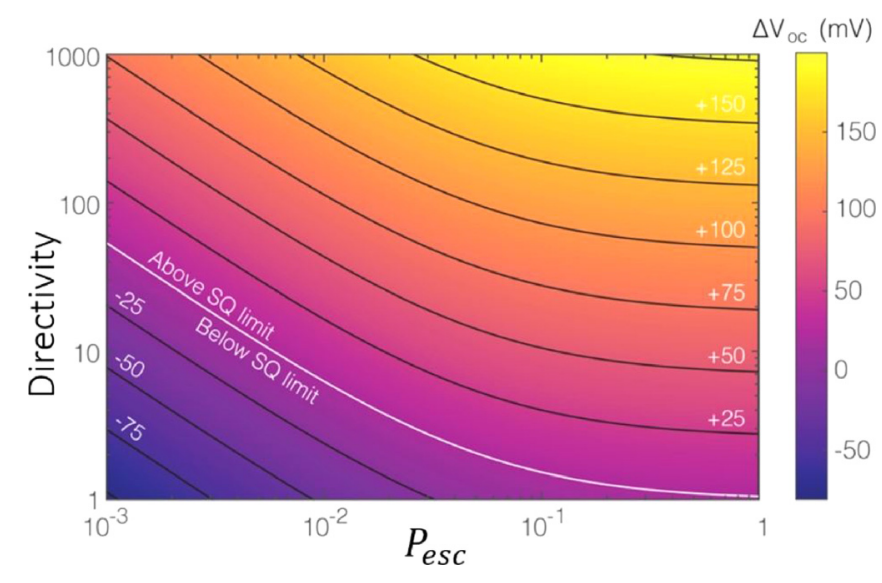

FIG. 22. Predicted enhancement ${ }^{26}$ of the $V_{o c}$ calculated with an internal photoluminescence efficiency $\eta_{\text {int }}^{\mathrm{PL}}=95 \%$ as a function of the photon escape probability $\mathrm{P}_{\mathrm{esc}}$ and the directivity of the external emission $\left(4 \pi / \varepsilon_{\text {emission }}\right)$. The Shockley-Queisser limit $\Delta V_{o c}=0$ is shown as a white curve. For a high directivity and reasonable $\mathrm{P}_{\mathrm{esc}}>0.1$, a sizeable increase in $\Delta V_{o c}$ above the S-Q limit is predicted. Reproduced with permission from Mann et al., ACS Nano 10, 8620-8631 (2016). Copyright 2016 American Chemical Society.

way. On the other hand, well-designed deterministic surface texturing might help to direct the emitted light back to the sun. In any case, the light path in an ultimate limit solar cell should be completely reversible in the spectral region where the photoluminescence is emitted. An ideal solar cell would, however, be capable to absorb diffuse light outside this spectral region, which will increase the short circuit current. In the ideal case, the absorbed diffusively scattered light will subsequently be emitted into a small solid angle, directed back to the sun. The consequences of the diffusively scattered light to the open circuit voltage are an interesting subject for further research.

At the present stage of nanowire solar cell research, the realization of an ultimate limit cell is still a dream. It is, however, important to gather more knowledge on the influence of the internal photoluminescence efficiency, the photon extraction efficiency, and the directivity of the emitted light on the open circuit voltage. Mann et al. ${ }^{26}$ have performed such a study for a GaAs solar cell with a high internal photoluminescence efficiency of $\eta_{i n t}^{P L}=95 \%$, the result of which is summarized in Fig. 22. The paper of Mann et al. also shows that it is possible to beat the Shockley Queisser limit with a relatively low internal photoluminescence efficiency of $\eta_{i n t}^{P L}=1 \%$, combined with a directivity of 100 and a photon escape probability of $100 \%$. Nanophotonic engineering for obtaining a high photon escape probability and a high directivity is thus at least equally important as the improvement of the internal photoluminescence efficiency for approaching the ultimate limit.

\section{ECONOMICS OF NANOWIRE SOLAR CELLS}

In real world applications, silicon solar cells have by far the largest market penetration, while III/V solar cells are only being used for space applications and as multi-junction solar cells for concentrator photovoltaics. Nanostructured solar cells promise a combination of high efficiency and low cost, ${ }^{178}$ in particular, when novel growth methods such as aerotaxy ${ }^{179-181}$ would prove to yield high performance. We like to look at a nanowire solar cell as typically a $2 \mu \mathrm{m}$ thick film on a silicon ${ }^{58,182-186}$ or, e.g., a graphene ${ }^{187,188}$ substrate with a nanowire filling fraction ${ }^{14,68}$ between $10 \%$ and $20 \%$. Since a III/V substrate constitutes the major fraction of the material cost of a III/V solar cell, elimination of the expensive substrate will substantially reduce the costs, as has been shown by the company Alta Devices for planar GaAs solar cells. ${ }^{166}$ The small nanowire filling fraction further reduces material consumption. It has been shown that it is possible to use a substrate multiple times by embedding the nanowires into PDMS and peeling them off. ${ }^{167,189}$ The substrate can subsequently be cleaned and re-used for a second and third growth run. ${ }^{167}$ Alternatively, the substrate costs can be fully eliminated by using aerotaxy. ${ }^{179-181}$ Challenges with aerotaxy are to vertically align the nanowires into an array, to achieve sizable solar light absorption on a large area as well as to provide electrical contacts.

Another interesting option is to grow multi-junction nanowire solar cells ${ }^{60}$ or mixed tandem cells ${ }^{190-196,205}$ consisting of a nanowire cell on top of, e.g., a planar $\mathrm{Si}$ cell. The principle of a nanowire tandem cell has been first demonstrated by Heurlin et al., ${ }^{197}$ using InP for both the top and the bottom cell. Heurlin et al. demonstrated a $V_{o c}$ of $1.15 \mathrm{~V}$ for the tandem configuration, which is a $67 \%$ increase with respect to the single junction cell. The first nanowire tunnel diodes comprising different bandgap semiconductors have been demonstrated by Zeng et al. ${ }^{198}$ and Otnes et al., ${ }^{199}$ while a radial tunnel junctions have recently been demonstrated by Tizno et al. ${ }^{200}$ Although a multi-junction nanowire solar cell has not yet been realized

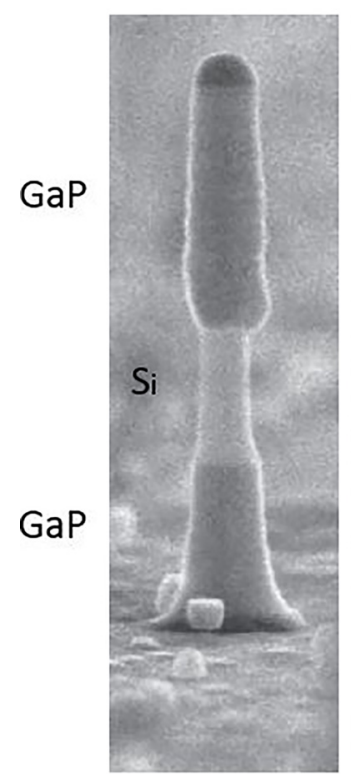

(a)

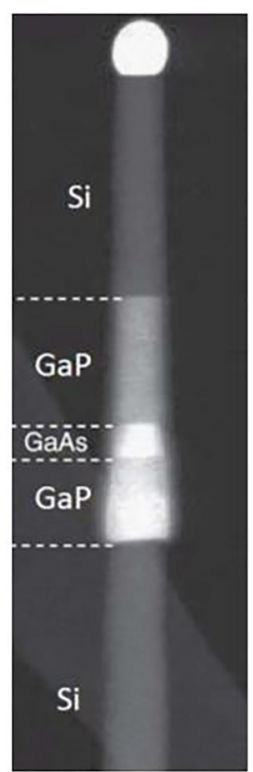

(b)

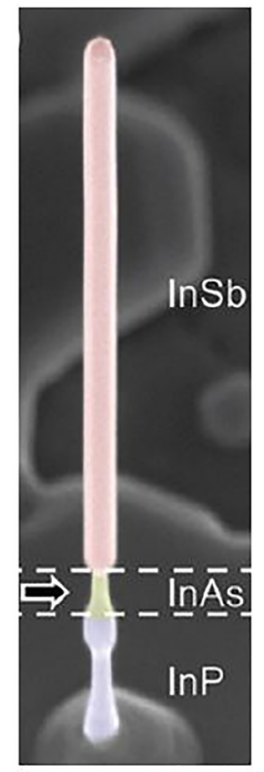

(c)
FIG. 23. Scanning electron micrographs of (a) an axial GaP-Si-GaP, (b) an axial Si-GaP-GaAs-GaP-Si, and (c) an axial InP-InAs-InSb heterojunction nanowire, suggesting the possibility to grow a multi-junction nanowire solar cell. Panels (a) and (b) are reproduced with permission from Hocevar et al., Nat. Commun. 3, 1266 (2012). Copyright 2012 Springer Nature. Panel (c) is reproduced with permission from Plissard et al., Nano Lett. 12, 1794-1798 (2012). Copyright 2012 American Chemical Society. 
experimentally, we like to emphasize that semiconductors with different lattice constants can be stacked into a single nanowire. In a single nanowire, the stacking of different bandgap semiconductors is possible without creating misfit dislocations due to the possibility of free radial expansion. As an example for the stacking of different semiconductors within a single nanowire, we show in Fig. 23(a) that a silicon section can be grown within a GaP nanowire and in Fig. 23(b) that a $\mathrm{GaP} / \mathrm{GaAs} / \mathrm{GaP}$ section can be grown within a Si nanowire. ${ }^{201,202}$ It is finally shown in Fig. 23(c) that one can start with a InP bottom section, followed by an InAs middle section to conclude with a reasonable long InSb nanowire. ${ }^{203}$ These results suggest that it is not impossible to grow a multi-junction nanowire solar cell in the future.

\section{CONCLUSIONS}

In the famous paper by Miller et al., ${ }^{22}$ it was stated that the optical design of a planar GaAs solar cell is of key importance for approaching the Shockley Queisser limiting efficiency. Nanowire solar cells feature much more possibilities to improve the optical design as compared to a planar solar cell. The important message of the present review is that nanophotonic engineering not only allows for maximizing the absorbance of the nanowire array aiming to optimize the short-circuit current of the solar cell. Nanophotonic engineering is also very promising to optimize the external radiative efficiency, which is the key metric for optimizing the open circuit voltage of the solar cell. Systematic work on the optimization of the external radiative efficiency of nanowire solar cells is largely lacking. It is, however, clear that a nanowire solar cell could fundamentally outperform its planar counterparts (for a realistic internal radiative efficiency) by optimizing the photon escape probability and thus achieving a higher external radiative efficiency than a planar solar cell. The key advantage of a nanowire solar cell is the fact that a large part of the emission is guided into a confined optical mode which can adiabatically expand into the air with only negligible reflection loss. It is also clear that a nanowire solar cell needs a highly reflective back mirror to avoid radiation losses in the underlying substrate.

Although nanowires feature many more degrees of freedom to tailor their nanophotonic properties compared to a planar layer, the disadvantage of the present generation of nanowires is their relatively poor internal radiative efficiency as compared to, e.g., a planar layer of GaAs. In bulk semiconductors, minority carrier lifetimes up to $14 \mu$ s have been observed $^{123}$ in double-heterostructure GaAs/InGaP and an internal radiative efficiency of $99.7 \%$ has been reported ${ }^{23}$ in a GaAs/AlGaAs double heterostructure. The highest reported minority carrier lifetime in a top-down etched $275 \mathrm{~nm}$ diameter nanopillar ${ }^{154}$ is presently limited to $22.8 \mathrm{~ns}$. Bottom-up grown nanowires presently feature minority carrier lifetimes below $10 \mathrm{~ns}$, which is three order of magnitude below their planar counterparts. We emphasize that we did not identify any fundamental reason for the low internal photoluminescence efficiency of the present generation of nanowire solar cells. The only argument might be that it is more cumbersome to combine a high internal photoluminescence efficiency of the bulk material with a high quality surface passivation like, e.g., an epitaxial shell. Progress in the optical quality of semiconductor nanowires is thus of utmost importance in order to further exploit advanced nanophotonic designs.

Once it would be possible to reach a high internal radiative efficiency, nanowire solar cells might become the primary vehicle to exploit the yet unexplored ultimate limit by nanophotonic engineering. The key advantage of a nanowire design is that the light propagation is highly deterministic, since it is connected with the guided optical mode of the nanowire optical cavity. We anticipate that nanowires will allow to reduce the entropy loss of a solar cell by focusing the emitted light (partly) back to the sun. Nanophotonic engineering of the entropy loss of the light is expected to become an important field of research, in particular, when this research would also consider the entropy losses due to light scattering in the atmosphere. We are convinced that the ultimate solar cell concept is moving solar cell research into a completely unexplored territory where both theoretical and experimental works are urgently required.

\section{ACKNOWLEDGMENTS}

We would like to thank all people involved in the work, especially Dick van Dam, Sander Mann, Yingchao Cui, Alessandro Cavalli, Sebastian Oener, Rene van Veldhoven, Thuy Vu, Anthony Standing, Niels van Hoof, Lachlan Black, and Professor Jaime Gomez Rivas for their invaluable contribution. We thank Dick van Dam for Figs. 1 and 7. This work was part of the research program of NWO-ENW and NWO-TTW with Project Nos. STW11826, FOM16PR1043, TTW15971, and 739.017.002, which was (partly) financed by The Netherlands Organization for Scientific Research (NWO).

${ }^{1}$ B. Tian, T. J. Kempa, and C. M. Lieber, "Single nanowire photovoltaics," Chem. Soc. Rev. 38, 16-24 (2009).

${ }^{2}$ E. C. Garnett, M. L. Brongersma, Y. Cui, and M. D. McGehee, "Nanowire solar cells," Annu. Rev. Mater. Res. 41, 269-295 (2011).

${ }^{3}$ T. J. Kempa, R. W. Day, S.-K. Kim, H.-G. Park, and C. M. Lieber, "Semiconductor nanowires: A platform for exploring limits and concepts for nano-enabled solar cells," Energy Environ. Sci. 6, 719 (2013).

${ }^{4}$ T. J. Kempa et al., "Coaxial multishell nanowires with high-quality electronic interfaces and tunable optical cavities for ultrathin photovoltaics," Proc. Natl. Acad. Sci. U. S. A. 109, 1407-1412 (2012).

${ }^{5}$ R. R. Lapierre et al., "III-V nanowire photovoltaics: Review of design for high efficiency," Phys. Status Solidi RRL 7, 815-830 (2013).

${ }^{6} \mathrm{~S}$. Mokkapati and C. Jagadish, "Review on photonic properties of nanowires for photovoltaics, [Invited]," Opt. Express 24, 17345 (2016).

${ }^{7}$ N. M. Ali and N. H. Rafat, "Modeling and simulation of nanorods photovoltaic solar cells: A review," Renewable Sustainable Energy Rev. 68, 212-220 (2017).

${ }^{8}$ G. Otnes and M. T. Borgström, "Towards high efficiency nanowire solar cells," Nano Today 12, 31-45 (2017).

${ }^{9} \mathrm{P}$. Yu et al., "Design and fabrication of silicon nanowires towards efficient solar cells," Nano Today 11, 704-737 (2016).

${ }^{10}$ W. Shockley and H. J. Queisser, "Detailed balance limit of efficiency of p-n junction solar cells,” J. Appl. Phys. 32, 510-519 (1961).

${ }^{11}$ H. J. Queisser, "Detailed balance limit for solar cell efficiency," Mater. Sci. Eng., B 159-160, 322-328 (2009). 
${ }^{12}$ A. Polman, M. Knight, E. C. Garnett, B. Ehrler, and W. C. Sinke, "Photovoltaic materials: Present efficiencies and future challenges," Science 352, 4424 (2016).

${ }^{13}$ M. A. Green and S. P. Bremner, "Energy conversion approaches and materials for high-efficiency photovoltaics," Nat. Mater. 16, 23-34 (2017).

${ }^{14} \mathrm{~J}$. Wallentin et al., "InP nanowire array solar cells achieving $13.8 \%$ efficiency by exceeding the ray optics limit," Science 339, 1057-1060 (2013).

${ }^{15}$ I. Aberg et al., "A GaAs nanowire array solar cell with $15.3 \%$ efficiency at 1 sun," IEEE J. Photovoltaics 6, 185-190 (2016).

${ }^{16}$ D. Van Dam et al., "High-efficiency nanowire solar cells with omnidirectionally enhanced absorption due to self-aligned indium-tin-oxide Mie scatterers," ACS Nano 10, 11414-11419 (2016).

${ }^{17} \mathrm{~S}$. Rühle, "Tabulated values of the Shockley-Queisser limit for single junction solar cells," Sol. Energy 130, 139-147 (2016).

${ }^{18}$ U. Rau, U. W. Paetzold, and T. Kirchartz, "Thermodynamics of light management in photovoltaic devices," Phys. Rev. B: Condens. Matter Mater. Phys. 90, 35211 (2014).

${ }^{19} \mathrm{P}$. Krogstrup et al., "Single-nanowire solar cells beyond the Shockley-Queisser limit," Nat. Photonics 7, 306-310 (2013).

${ }^{20} \mathrm{~S}$. Sandhu, Z. Yu, and S. Fan, "Detailed balance analysis and enhancement of open-circuit voltage in single-nanowire solar cells," Nano Lett. 14, 1011-1015 (2014)

${ }^{21}$ S. A. Mann et al., "Quantifying losses and thermodynamic limits in nanophotonic solar cells," Nat. Nanotechnol. 11, 1071-1075 (2016).

${ }^{22}$ O. D. Miller, E. Yablonovitch, and S. R. Kurtz, "Strong internal and external luminescence as solar cells approach the Shockley-Queisser limit," IEEE J. Photovoltaics 2, 303-311 (2012).

${ }^{23}$ I. Schnitzer, E. Yablonovitch, C. Caneau, and T. J. Gmitter, "Ultrahigh spontaneous emission quantum efficiency, 99.7\% internally and $72 \%$ externally, from AlGaAs/GaAs/AlGaAs double heterostructures," Appl. Phys. Lett. 62, 131-133 (1993).

${ }^{24}$ O. Semyonov, A. Subashiev, Z. Chen, and S. Luryi, "Radiation efficiency of heavily doped bulk n-InP semiconductor," J. Appl. Phys. 108, 013101 (2010).

${ }^{25}$ J. F. Geisz et al., "Enhanced external radiative efficiency for $20.8 \%$ efficient single-junction GaInP solar cells," Appl. Phys. Lett. 103, 41118 (2013).

${ }^{26}$ S. A. Mann, R. R. Grote, R. M. Osgood, A. Alù, and E. C. Garnett, "Opportunities and limitations for nanophotonic structures to exceed the Shockley-Queisser limit," ACS Nano 10, 8620-8631 (2016).

${ }^{27}$ S. Sandhu, Z. Yu, and S. Fan, "Detailed balance analysis of nanophotonic solar cells," Opt. Express 21, 1209 (2013).

${ }^{28}$ W. Van Roosbroeck and W. Shockley, "Photon-radiative recombination of electrons and holes in germanium," Phys. Rev. 94, 1558-1560 (1954).

${ }^{29} \mathrm{U}$. Rau and T. Kirchartz, "On the thermodynamics of light trapping in solar cells," Nat. Mater. 13, 103-104 (2014).

${ }^{30}$ R. T. Ross, "Some thermodynamics of photochemical systems," J. Chem. Phys. 46, 4590-4593 (1967).

${ }^{31} \mathrm{G}$. Smestad and H. Ries, "Luminescence and current-voltage characteristics of solar cells and optoelectronic devices," Sol. Energy Mater. Sol. Cells 25, 51-71 (1992).

${ }^{32} \mathrm{U}$. Rau, "Reciprocity relation between photovoltaic quantum efficiency and electroluminescent emission of solar cells," Phys. Rev. B: Condens. Matter Mater. Phys. 76, 85303 (2007).

${ }^{33} \mathrm{U}$. Rau, "Superposition and reciprocity in the electroluminescence and photoluminescence of solar cells," IEEE J. Photovoltaics 2, 169-172 (2012).

${ }^{34}$ Y. Cui et al., "Boosting solar cell photovoltage via nanophotonic engineering," Nano Lett. 16, 6467-6471 (2016).

${ }^{35}$ V. Ganapati, M. A. Steiner, and E. Yablonovitch, "The voltage boost enabled by luminescence extraction in solar cells," IEEE J. Photovoltaics 6, 801-809 (2016).

${ }^{36}$ Z. Yu, S. Sandhu, and S. Fan, "Efficiency above the ShockleyQueisser limit by using nanophotonic effects to create multiple effective bandgaps with a single semiconductor," Nano Lett. 14, 66-70 (2014).

${ }^{37}$ M. Law, L. E. Greene, J. C. Johnson, R. Saykally, and P. Yang, "Nanowire dye-sensitized solar cells," Nat. Mater. 4, 455-459 (2005).

${ }^{38}$ R. Yan, D. Gargas, and P. Yang, "Nanowire photonics," Nat. Photonics 3, 569-576 (2009).
${ }^{39}$ E. C. Garnett and P. Yang, "Silicon nanowire radial p-n junction solar cells,” J. Am. Chem. Soc. 130, 9224-9225 (2008).

${ }^{40}$ M. Law, J. Goldberger, and P. Yang, "Semiconductor nanowires and nanotubes," Annu. Rev. Mater. Res. 34, 83-122 (2004).

${ }^{41} \mathrm{~B}$. Tian et al., "Coaxial silicon nanowires as solar cells and nanoelectronic power sources," Nature 449, 885-889 (2007).

${ }^{42}$ T. J. Kempa et al., "Single and tandem axial p-i-n nanowire photovoltaic devices," Nano Lett. 8, 3456-3460 (2008).

${ }^{43} \mathrm{~L}$. Tsakalakos, "Strong broadband optical absorption in silicon nanowire films," J. Nanophotonics 1, 13552 (2007).

${ }^{44}$ L. Tsakalakos et al., "Silicon nanowire solar cells," Appl. Phys. Lett. 91, 233117 (2007).

${ }^{45}$ I. Gur, N. A. Fromer, M. L. Geier, and A. P. Alivisatos, "Air-stable allinorganic nanocrystal solar cells processed from solution," Science 310, 462-466 (2005).

${ }^{46} \mathrm{H}$. Goto et al., "Growth of core-shell inP nanowires for photovoltaic application by selective-area metal organic vapor phase epitaxy," Appl. Phys. Express 2, 035004 (2009).

${ }^{47} \mathrm{P}$. Mohan, J. Motohisa, and T. Fukui, "Controlled growth of highly uniform, axial/radial direction-defined, individually addressable InP nanowire arrays," Nanotechnology 16, 2903-2907 (2005).

${ }^{48}$ M. Yoshimura, E. Nakai, K. Tomioka, and T. Fukui, "Indium phosphide core-shell nanowire array solar cells with lattice-mismatched window layer," Appl. Phys. Express 6, 052301 (2013).

${ }^{49}$ M. Yoshimura, E. Nakai, K. Tomioka, and T. Fukui, "Indium tin oxide and indium phosphide heterojunction nanowire array solar cells," Appl. Phys. Lett. 103, 243111 (2013)

${ }^{50} \mathrm{Y}$. Kim et al., "Influence of nanowire density on the shape and optical properties of ternary InGaAs nanowires," Nano Lett. 6, 599-604 (2006).

${ }^{51} \mathrm{H}$. J. Joyce et al., "High purity GaAs nanowires free of planar defects: Growth and characterization," Adv. Funct. Mater. 18, 3794-3800 (2008).

${ }^{52} \mathrm{H}$. J. Joyce et al., "Unexpected benefits of rapid growth rate for III-V nanowires," Nano Lett. 9, 695-701 (2008).

${ }^{53} \mathrm{P}$. Parkinson et al., "Carrier lifetime and mobility enhancement in nearly defect-free core- shell nanowires measured using time-resolved terahertz spectroscopy," Nano Lett. 9, 3349-3353 (2009).

${ }^{54}$ B. M. Kayes, H. A. Atwater, and N. S. Lewis, "Comparison of the device physics principles of planar and radial p-n junction nanorod solar cells," J. Appl. Phys. 97, 114302 (2005).

${ }^{55}$ M. D. Kelzenberg et al., "Photovoltaic measurements in single-nanowire Si SCs," Nano Lett. 8, 710-714 (2008).

${ }^{56}$ T. Mårtensson, P. Carlberg, M. Borgstrom, L. Montelius, W. Seifert, and L. Samuelson, "Nanowire arrays defined by nanoimprint lithography," Nano Lett. 4, 699-702 (2004).

${ }^{57}$ M. T. Björk et al., "Nanowire resonant tunneling diodes," Appl. Phys. Lett. 81, 4458-4460 (2002).

${ }^{58}$ T. Mårtensson et al., "Epitaxial III-V nanowires on silicon," Nano Lett. 4, 1987-1990 (2004).

${ }^{59} \mathrm{~N}$. Sköld et al., "Growth and optical properties of strained GaAs- $\mathrm{Ga}_{\mathrm{x}} \mathrm{In}_{1-\mathrm{x}} \mathrm{P}$ core-shell nanowires," Nano Lett. 5, 1943-1947 (2005).

${ }^{60}$ A. Kandala, T. Betti, and A. Fontcuberta, "General theoretical considerations on nanowire solar cell designs," Phys. Status Solidi A 206, 173-178 (2009).

${ }^{61} \mathrm{~J}$. Dufouleur et al., "P-doping mechanisms in catalyst-free gallium arsenide nanowires," Nano Lett. 10, 1734-1740 (2010).

${ }^{62} \mathrm{X}$. Li and Y. Zhan, "Enhanced external quantum efficiency in rectangular single nanowire solar cells," Appl. Phys. Lett. 102, 21101 (2013).

${ }^{63}$ M. Heiss and A. Fontcuberta I Morral, "Fundamental limits in the external quantum efficiency of single nanowire solar cells," Appl. Phys. Lett. 99, 263102 (2011).

${ }^{64}$ J. Zhu, C. M. Hsu, Z. Yu, S. Fan, and Y. Cui, "Nanodome solar cells with efficient light management and self-cleaning," Nano Lett. 10, 1979-1984 (2010).

${ }^{65} \mathrm{~J}$. Zhu et al., "Optical absorption enhancement in amorphous silicon nanowire and nanocone arrays," Nano Lett. 9, 279-282 (2009).

${ }^{66}$ T. Stelzner et al., "Silicon nanowire-based solar cells," Nanotechnology 19, 295203 (2008).

${ }^{67}$ O. Gunawan and S. Guha, "Characteristics of vapor-liquid-solid grown silicon nanowire solar cells," Sol. Energy Mater. Sol. Cells 93, 1388-1393 (2009).

${ }^{68} \mathrm{Y}$. Cui et al., "Efficiency enhancement of InP nanowire solar cells by surface cleaning," Nano Lett. 13, 4113-4117 (2013). 
${ }^{69}$ J. V. Holm et al., "Surface-passivated GaAsP single-nanowire solar cells exceeding 10\% efficiency grown on silicon," Nat. Commun. 4, 1498 (2013).

${ }^{70}$ M. Aagesen, J. V. Holm, H. I. Jorgensen, and H. Liu, "GaAsP single nanowire solar cells grown on silicon exhibiting large Voc increase at multiple suns," in Proceedings of IEEE Photovolt. Spec. Conf. (2013), pp. 254-257.

${ }^{71}$ Y. Xu, T. Gong, and J. N. Munday, "The generalized Shockley-Queisser limit for nanostructured solar cells," Sci. Rep. 5, 13536 (2015).

${ }^{72}$ G. L. Araujo and A. Marti, "Absolute limiting efficiencies for photovoltaic energy-conversion," Sol. Energy Mater. Sol. Cells 33, 213-240 (1994).

${ }^{73}$ A. Martí, J. L. Balenzategui, and R. F. Reyna, "Photon recycling and Shockley's diode equation,” J. Appl. Phys. 82, 4067-4075 (1997).

${ }^{74} \mathrm{~N}$. Anttu, "Shockley-Queisser detailed balance efficiency limit for nanowire solar cells," ACS Photonics 2, 446-453 (2015).

${ }^{75}$ O. L. Muskens, J. G. Rivas, R. E. Algra, E. P. A. M. Bakkers, and A. Lagendijk, "Design of light scattering in nanowire materials for photovoltaic applications," Nano Lett. 8, 2638-2642 (2008).

${ }^{76} \mathrm{~S}$. Hu et al., "Optical, electrical, and solar energy-conversion properties of gallium arsenide nanowire-array photoanodes," Energy Environ. Sci. 6, 1879 (2013)

${ }^{77} \mathrm{~L}$. Hu and G. Chen, "Analysis of optical absorption in silicon nanowire arrays for photovoltaic applications," Nano Lett. 7, 3249-3252 (2007).

${ }^{78} \mathrm{~N}$. Anttu and H. Q. Xu, "Efficient light management in vertical nanowire arrays for photovoltaics," Opt. Express 21, A558 (2013).

${ }^{79} \mathrm{~N}$. Anttu and H. Q. Xu, "Coupling of light into nanowire arrays and subsequent absorption," J. Nanosci. Nanotechnol. 10, 7183-7187 (2010).

${ }^{80} \mathrm{~N}$. Anttu, "Geometrical optics, electrostatics, and nanophotonic resonances in absorbing nanowire arrays," Opt. Lett. 38, 730-732 (2013).

${ }^{81}$ J. Kupec, R. L. Stoop, and B. Witzigmann, "Light absorption and emission in nanowire array solar cells," Opt. Express 18, 27589-27605 (2010).

${ }^{82}$ Y. Hu, R. R. Lapierre, M. Li, K. Chen, and J. J. He, "Optical characteristics of GaAs nanowire solar cells," J. Appl. Phys. 112, 104311 (2012).

${ }^{83}$ J. A. Czaban, D. A. Thompson, and R. R. LaPierre, "GaAs core-shell nanowires for photovoltaic applications," Nano Lett. 9, 148-154 (2009).

${ }^{84}$ R. R. Lapierre, "Numerical model of current-voltage characteristics and efficiency of GaAs nanowire solar cells," J. Appl. Phys. 109, 034311 (2011).

${ }^{85}$ A. C. E. Chia et al., "Electrical transport and optical model of GaAsAlInP core-shell nanowires," J. Appl. Phys. 111, 094319 (2012).

${ }^{86} \mathrm{C}$. Gutsche et al., "N-GaAs/InGaP/p-GaAs core-multishell nanowire diodes for efficient light-to-current conversion,” Adv. Funct. Mater. 22, 929-936 (2012).

${ }^{87} \mathrm{C}$. Gutsche et al., "Controllable p-type doping of GaAs nanowires during vapor-liquid-solid growth,” J. Appl. Phys. 105, 24305 (2009).

${ }^{88} \mathrm{C}$. Gutsche et al., "n-type doping of vapor-liquid-solid grown GaAs nanowires," Nanoscale Res. Lett. 6, 1-6 (2011).

${ }^{89} \mathrm{P}$. Kailuweit et al., "Numerical simulations of absorption properties of InP nanowires for solar cell applications," Prog. Photovoltaics 20, 945-953 (2012).

${ }^{90} \mathrm{M}$. Yao et al., "GaAs nanowire array solar cells with axial p-i-n junctions," Nano Lett. 14, 3293-3303 (2014).

${ }^{91} \mathrm{G}$. Jia et al., "Silicon nanowire solar cells with radial p-n heterojunction on crystalline silicon thin films: Light trapping properties," IEEE J. Photovoltaics 4, 28-32 (2014).

${ }^{92}$ Y. Yu, V. E. Ferry, A. P. Alivisatos, and L. Cao, "Dielectric core-shell optical antennas for strong solar absorption enhancement," Nano Lett. 12, 3674-3681 (2012).

${ }^{93} \mathrm{Z}$. Zhong et al., "Efficiency enhancement of axial junction InP single nanowire solar cells by dielectric coating," Nano Energy 28, 106-114 (2016).

${ }^{94}$ X. Wang, M. R. Khan, M. Lundstrom, and P. Bermel, "Performance-limiting factors for GaAs-based single nanowire photovoltaics," Opt. Express 22, A344 (2014).

${ }^{95}$ M. Heiss, "III - V nanowire arrays: Growth and light interaction," Nanotechnology 25, 14015 (2014).

${ }^{96}$ K. T. Fountaine, W. H. Cheng, C. R. Bukowsky, and H. A. Atwater, "Near-unity unselective absorption in sparse InP nanowire arrays," ACS Photonics 3, 1826-1832 (2016).
${ }^{97}$ G. Otnes et al., Understanding InP nanowire array solar cell performance by nanoprobe-enabled single nanowire measurements. 18, 3038-3046 (2018).

${ }^{98}$ Y. Chen, P. Kivisaari, M. E. Pistol, and N. Anttu, "Optimization of the short-circuit current in an InP nanowire array solar cell through optoelectronic modeling," Nanotechnology 27, 435404 (2016).

${ }^{99}$ S. L. Diedenhofen, O. T. A. Janssen, G. Grzela, E. P. A. M. Bakkers, and J. Gómez Rivas, "Strong geometrical dependence of the absorption of light in arrays of semiconductor nanowires," ACS Nano 5, 2316-2323 (2011).

${ }^{100}$ M. L. Brongersma, Y. Cui, and S. Fan, "Light management for photovoltaics using high-index nanostructures," Nat. Mater. 13, 451-460 (2014).

${ }^{101}$ L. Cao et al., "Semiconductor nanowire optical antenna solar absorbers," Nano Lett. 10, 439-445 (2010).

${ }^{102}$ L. Cao, P. Fan, and M. L. Brongersma, "Optical coupling of deepsubwavelength semiconductor nanowires," Nano Lett. 11, 1463-1468 (2011).

${ }^{103} \mathrm{~L}$. Cao et al., "Engineering light absorption in semiconductor nanowire devices," Nat. Mater. 8, 643-647 (2009).

${ }^{104}$ D. Van Dam et al., "Directional and polarized emission from nanowire arrays," Nano Lett. 15, 4557-4563 (2015).

${ }^{105} \mathrm{~N}$. Anttu et al., "Absorption of light in InP nanowire arrays," Nano Res. 7, 816-823 (2014).

${ }^{106}$ K. Seo et al., "Multicolored vertical silicon nanowires," Nano Lett. 11, 1851-1856 (2011).

${ }^{107} \mathrm{~B}$. Wang and P. W. Leu, "Tunable and selective resonant absorption in vertical nanowires," Opt. Lett. 37, 3756 (2012).

${ }^{108} \mathrm{~J}$.-H. Yun et al., "Incident light adjustable solar cell by periodic nanolens architecture," Sci. Rep. 4, 6879 (2014).

${ }^{109}$ O. M. Ghahfarokhi, N. Anttu, L. Samuelson, and I. Åberg, "Performance of GaAs nanowire array solar cells for varying incidence angles," IEEE J. Photovoltaics 6, 1502 (2016)

${ }^{110}$ M. A. Green, Y. Hishikawa, A. W. Y. H. Baillie, E. D. Dunlop, and D. H. Levi, "Solar cell efficiency tables (version 51)," Prog. Photovoltaics 26, 3-12 (2018).

${ }^{111}$ M. W. Wanlass, "System and methods for advanced ultra-high-performance InP solar cells," U.S. patent 0,280,042 (2015), p. 16.

${ }^{112} \mathrm{H}$. Schimper et al., "Material studies regarding InP-based high-efficiency solar cells," J. Cryst. Growth 287, 642-646 (2006).

${ }^{113}$ R. J. Nelson and R. G. Sobers, "Minority-carrier lifetimes and internal quantum efficiency of surface-free GaAs," J. Appl. Phys. 49, 6103-6108 (1978).

${ }^{114}$ B. Ullrich, S. R. Munshi, and G. J. Brown, "Photoluminescence analysis of p-doped GaAs using the Roosbroeck-Shockley relation," Semicond. Sci. Technol. 22, 1174-1177 (2007).

${ }^{115} \mathrm{E}$. Yablonovitch, T. J. Gmitter, and R. Bhat, "Inhibited and enhanced spontaneous emission from optically thin Al GaAs GaAs double heterostructures," Phys. Rev. Lett. 61, 2546-2549 (1988).

${ }^{116}$ R. K. Ahrenkiel et al., "Recombination lifetimes in undoped, low-band gap InAsyP $\mathrm{P}_{1-\mathrm{y}} / \mathrm{In}_{\mathrm{x}} \mathrm{Ga}_{1-\mathrm{x}} \mathrm{As}$ double heterostructures grown on InP substrates," Appl. Phys. Lett. 78, 1092-1094 (2001).

${ }^{117}$ G. B. Lush, "B-coefficient in n-type GaAs," Sol. Energy Mater. Sol. Cells 93, 1225-1229 (2009).

${ }^{118}$ Y. Rosenwaks, I. Tsimberova, H. Gero, and M. Molotskii, "Minority-carrier recombination in p-InP single crystals," Phys. Rev. B 68, 115210 (2003).

${ }^{119}$ I. Tsimberova, Y. Rosenwaks, and M. Molotskii, "Minority carriers recombination in n-InP single crystals," J. Appl. Phys. 93, 9797-9802 (2003).

${ }^{120}$ Y. Rosenwaks, Y. Shapira, and D. Huppert, "Picosecond time-resolved luminescence of surface and bulk recombination processes in InP," Phys. Rev. B 45, 9108-9119 (1992).

${ }^{121}$ L. W. Molenkamp, G. L. M. Kampschöer, W. De Lange, J. W. F. M. Maes, and P. J. Roksnoer, "Ultralong minority-carrier lifetimes in GaAs grown by low-pressure organometallic vapor phase epitaxy," Appl. Phys. Lett. 54, 1992-1994 (1989).

${ }^{122}$ R. K. Ahrenkiel et al., "Ultralong minority-carrier lifetime epitaxial GaAs by photon recycling," Appl. Phys. Lett. 55, 1088-1090 (1989).

${ }^{123}$ J. M. Olson, R. K. Ahrenkiel, D. J. Dunlavy, B. Keyes, and A. E. Kibbler, "Ultralow recombination velocity at $\mathrm{Ga}_{0.5} \mathrm{In}_{0.5} \mathrm{P} / \mathrm{GaAs}$ heterointerfaces," Appl. Phys. Lett. 55, 1208-1210 (1989).

${ }^{124}$ R. K. Ahrenkiel, R. Ellingson, S. Johnston, and M. Wanlass, "Recombination lifetime of InGaAs as a function of doping density," Appl. Phys. Lett. 72, 3470-3472 (1998). 
${ }^{125}$ G. W. Hooft and C. Van Opdorp, "Temperature dependence of interface recombination and radiative recombination in ( $\mathrm{Al}, \mathrm{Ga})$ As heterostructures," Appl. Phys. Lett. 42, 813 (1983).

${ }^{126}$ G. W. 'tHooft, M. R. Leys, and H. J. Talen-vd Mheen, “Temperature dependence of the radiative recombination coefficient in GaAs - (Al, Ga)As quantum wells," Superlattices Microstruct. 1, 307-310 (1985).

${ }^{127}$ Y. Rosenwaks, Y. Shapira, and D. Huppert, "Evidence for low intrinsic surface-recombination velocity on p-type InP," Phys. Rev. B 44, 13097-13100 (1991).

${ }^{128} \mathrm{~W}$. Zhang et al., "Carrier recombination dynamics in sulfur-doped InP nanowires," Nano Lett. 15, 7238-7244 (2015).

${ }^{129} \mathrm{~W}$. Zhang et al., "Carrier recombination processes in gallium indium phosphide nanowires," Nano Lett. 17, 4248-4254 (2017).

${ }^{130}$ H. J. Joyce et al., "Ultralow surface recombination velocity in InP nanowires probed by terahertz spectroscopy," Nano Lett. 12, 5325-5330 (2012).

${ }^{131}$ A. Cavalli et al., "Influence of growth conditions on the performance of InP nanowire solar cells," Nanotechnology 27, 454003 (2016).

${ }^{132}$ T. T. T. Vu et al., "High optical quality single crystal phase wurtzite and zincblende InP nanowires," Nanotechnology 24, 115705 (2013).

${ }^{133}$ L. E. Black et al., "Effective surface passivation of InP nanowires by atomic-layer-deposited $\mathrm{A} 12 \mathrm{O} 3$ with POx interlayer," Nano Lett. 17, 6287-6294 (2017).

${ }^{134}$ D. Tedeschi et al., "Long-lived hot carriers in III-V nanowires," Nano Lett. 16, 3085-3093 (2016).

${ }^{135} \mathrm{Q}$. Gao et al., "Selective-area epitaxy of pure wurtzite InP nanowires: High quantum efficiency and room-temperature lasing," Nano Lett. 14, 5206-5211 (2014)

${ }^{136} \mathrm{~K}$. Li et al., "Wurtzite-phased InP micropillars grown on silicon with low surface recombination velocity," Nano Lett. 15, 7189-7198 (2015).

${ }^{137} \mathrm{~F}$. Wang et al., "Spatially resolved doping concentration and nonradiative lifetime profiles in single Si-doped InP nanowires using photoluminescence mapping," Nano Lett. 15, 3017-3023 (2015).

${ }^{138}$ T. T. D. Tran et al., "High brightness InP micropillars grown on silicon with Fermi level splitting larger than 1 eV," Nano Lett. 14, 3235-3240 (2014).

${ }^{139}$ F. Ren, K. Wei Ng, K. Li, H. Sun, and C. J. Chang-Hasnain, "High-quality InP nanoneedles grown on silicon," Appl. Phys. Lett. 102, 012115 (2013).

${ }^{140}$ H. J. Joyce, J. Wong-Leung, Q. Gao, H. Hoe Tan, and C. Jagadish, "Phase perfection in zinc blende and wurtzite III-V nanowires using basic growth parameters," Nano Lett. 10, 908-915 (2010).

${ }^{141}$ C. Colombo et al., "Gallium arsenide radial structures for photovoltaic applications,” Appl. Phys. Lett. 94, 173108 (2009).

${ }^{142}$ A. Fontcuberta i Morral, C. Colombo, G. Abstreiter, J. Arbiol, and J. R. Morante, "Nucleation mechanism of gallium-assisted molecular beam epitaxy growth of gallium arsenide nanowires Nucleation mechanism of gallium-assisted molecular beam epitaxy growth of gallium arsenide nanowires," Appl. Phys. Lett. 92, 63112 (2008).

${ }^{143}$ B. Ketterer, E. Uccelli, and A. Fontcuberta i Morral, "Mobility and carrier density in p-type GaAs nanowires measured by transmission Raman spectroscopy," Nanoscale 4, 1789-1793 (2012).

${ }^{144} \mathrm{~J}$. L. Boland et al., "Increased photoconductivity lifetime in GaAs nanowires by controlled n-type and p-type doping," ACS Nano 10, 4219-4227 (2016).

${ }^{145}$ C.-C. Chang et al., "Electrical and optical characterization of surface passivation in GaAs nanowires," Nano Lett. 12, 4484-4489 (2012).

${ }^{146} \mathrm{~N}$. Jiang et al., "Enhanced minority carrier lifetimes in GaAs/AlGaAs core-shell nanowires through shell growth optimization," Nano Lett. 13, 5135-5140 (2013).

${ }^{147}$ N. Jiang et al., "Long minority carrier lifetime in Au-catalyzed GaAs/ $\mathrm{Al}_{\mathrm{x}} \mathrm{Ga}_{1-\mathrm{x}} \mathrm{As}$ core-shell nanowires," Appl. Phys. Lett. 101, 23111 (2012).

${ }^{148}$ S. L. Chen, W. M. Chen, F. Ishikawa, and I. A. Buyanova, "Suppression of non-radiative surface recombination by $\mathrm{N}$ incorporation in GaAs/ GaNAs core/shell nanowires," Sci. Rep. 5, 11653 (2015).

${ }^{149}$ C. K. Yong et al., "Strong carrier lifetime enhancement in GaAs nanowires coated with semiconducting polymer," Nano Lett. 12, 6293-6301 (2012).

${ }^{150} \mathrm{O}$. Demichel et al., "Impact of surfaces on the optical properties of GaAs nanowires impact of surfaces on the optical properties of GaAs nanowires," Appl. Phys. Lett. 97, 201907 (2010).

${ }^{151} \mathrm{M}$. Hetzl et al., "Surface passivation and self-regulated shell growth in selective area-grown GaN-(Al,Ga)N core-shell nanowires," Nanoscale 9, 7179-7188 (2017).
${ }^{152} \mathrm{~S}$. Furthmeier et al., "Long exciton lifetimes in stacking-fault-free wurtzite GaAs nanowires," Appl. Phys. Lett. 105, 222109 (2014).

${ }^{153} \mathrm{~S}$. Breuer et al., "Suitability of Au- and self-assisted GaAs nanowires for optoelectronic applications," Nano Lett. 11, 1276-1279 (2011).

${ }^{154}$ A. Higuera-Rodriguez et al., "Ultralow surface recombination velocity in passivated InGaAs/InP nanopillars," Nano Lett. 17, 2627-2633 (2017).

${ }^{155} \mathrm{~T}$. Burgess et al., "Doping-enhanced radiative efficiency enables lasing in unpassivated GaAs nanowires," Nat. Commun. 7, 11927 (2016).

${ }^{156} \mathrm{X}$. Yin et al., " $19.2 \%$ efficient InP heterojunction solar cell with electronselective $\mathrm{TiO}_{2}$ contact," ACS Photonics 1, 1245-1250 (2014).

${ }^{157}$ C. Battaglia et al., "Hole selective $\mathrm{MoO}_{\mathrm{x}}$ contact for silicon solar cells," Nano Lett. 14, 967-971 (2014).

${ }^{158} \mathrm{C}$. Battaglia et al., "Silicon heterojunction solar cell with passivated hole selective $\mathrm{MoO}_{\mathrm{x}}$ contact,” Appl. Phys. Lett. 104, 113902 (2014).

${ }^{159}$ T. Kirchartz, F. Staub, and U. Rau, "Impact of photon recycling on the open-circuit voltage of metal halide perovskite solar cells," ACS Energy Lett. 1, 731-739 (2016).

${ }^{160}$ I. Friedler et al., "Solid-state single photon sources: The nanowire antenna," Opt. Express 17, 2095 (2009).

${ }^{161}$ M. E. Reimer et al., "Bright single-photon sources in bottom-up tailored nanowires," Nat. Commun. 3, 737 (2012).

${ }^{162}$ S. W. Schmitt, G. Sarau, and S. Christiansen, "Observation of strongly enhanced photoluminescence from inverted cone-shaped silicon nanostructures," Sci. Rep. 5, 17089 (2015).

${ }^{163} \mathrm{P}$. Kivisaari, Y. Chen, and N. Anttu, "Emission enhancement, light extraction and carrier dynamics in InGaAs/GaAs nanowire arrays," Nano Futures 2, 15001 (2018).

${ }^{164}$ T. Schmidt, K. Lischka, and W. Zulehner, "Excitation-power dependence of the near-band-edge photoluminescence of semiconductors," Phys. Rev. B 45, 8989-8994 (1992).

${ }^{165}$ G. J. Bauhuis, P. Mulder, E. J. Haverkamp, J. C. C. M. Huijben, and J. J. Schermer, "26.1\% thin-film GaAs solar cell using epitaxial lift-off," Sol. Energy Mater. Sol. Cells 93, 1488-1491 (2009).

${ }^{166}$ B. M. Kayes et al., " $27.6 \%$ conversion efficiency, a new record for single-junction solar cells under 1 sun illumination," in Proceedings of 37th Photovolt. Spec. Conf. (2011), pp. 4-8.

${ }^{167}$ A. Cavalli, A. Dijkstra, J. E. M. Haverkort, and E. P. A. M. Bakkers, "Nanowire polymer transfer for enhanced performance and lower cost," Nano-Struct. Nano-Objects 16, 59-62 (2018).

${ }^{168}$ A. Polman and H. A. Atwater, "Photonic design principles for ultrahighefficiency photovoltaics," Nat. Mater. 11, 174-177 (2012).

${ }^{169}$ E. D. Kosten, J. H. Atwater, J. Parsons, A. Polman, and H. A. Atwater, "Highly efficient GaAs solar cells by limiting light emission angle," Light: Sci. Appl. 2, e45 (2013).

${ }^{170}$ L. C. Hirst and N. J. Ekins-Daukes, "Fundamental losses in solar cells," Prog. Photovoltaics 19, 286-293 (2011).

${ }^{171}$ T. Markvart, "The thermodynamics of optical etendue," J. Opt. A: Pure Appl. Opt. 10, 15008 (2008).

${ }^{172}$ T. Markvart, "Solar cell as a heat engine: Energy-entropy analysis of photovoltaic conversion," Phys. Status Solidi A 205, 2752-2756 (2008).

${ }^{173}$ M. Peters et al., "Directionally selective light trapping in a germanium solar cell," Opt. Express 19, A136 (2011).

${ }^{174}$ M. Peters, J. Christoph Goldschmidt, and B. Blsi, "Efficiency limit and example of a photonic solar cell," J. Appl. Phys. 110, 043104 (2011).

${ }^{175}$ O. Höhn, T. Kraus, U. T. Schwarz, and B. Bläsi, "Effects of angular confinement and concentration to realistic solar cells," J. Appl. Phys. 117, 034503 (2015).

${ }^{176}$ E. D. Kosten, B. K. Newman, J. V. Lloyd, A. Polman, and H. A. Atwater, "Limiting light escape angle in silicon photovoltaics: Ideal and realistic cells," IEEE J. Photovoltaics 5, 61-69 (2015).

${ }^{177}$ E. D. Kosten, B. M. Kayes, and H. A. Atwater, "Experimental demonstration of enhanced photon recycling in angle-restricted GaAs solar cells," Energy Environ. Sci. 7, 1907-1912 (2014).

${ }^{178}$ M. C. Beard, J. M. Luther, and A. J. Nozik, "The promise and challenge of nanostructured solar cells," Nat. Nanotechnol. 9, 951-954 (2014).

${ }^{179}$ M. Heurlin et al., "Continuous gas-phase synthesis of nanowires with tunable properties," Nature 492, 90-94 (2012).

${ }^{180} \mathrm{~W}$. Metaferia et al., "GaAsP nanowires grown by aerotaxy," Nano Lett. 16, 5701-5707 (2016).

${ }^{181}$ E. Barrigón et al., "GaAs nanowire pn-junctions produced by low-cost and high-throughput aerotaxy," Nano Lett. 18, 1088-1092 (2018). 
${ }^{182}$ A. L. Roest et al., "Position-controlled epitaxial $\{$ III-V $\}$ nanowires on silicon," Nanotechnology 17, S271 (2006).

${ }^{183} \mathrm{~K}$. Tomioka and T. Fukui, "Recent progress in integration of III-V nanowire transistors on Si substrate by selective-area growth," J. Phys. D: Appl. Phys. 47, 394001 (2014).

${ }^{184}$ J. P. Boulanger et al., "Characterization of a Ga-assisted GaAs nanowire array solar cell on Si substrate," IEEE J. Photovoltaics 6, 661-667 (2016).

${ }^{185}$ M. Borg et al., "Vertical III-V nanowire device integration on Si(100)," Nano Lett. 14, 1914-1920 (2014).

${ }^{186}$ F. Matteini, G. Tütüncüoglu, D. Rüffer, E. Alarcón-Lladó, and A. Fontcuberta I Morral, "Ga-assisted growth of GaAs nanowires on silicon, comparison of surface SiOx of different nature," J. Cryst. Growth 404, 246-255 (2014)

${ }^{187}$ A. M. Munshi et al., "Vertically aligned GaAs nanowires on graphite and few-layer graphene: Generic model and epitaxial growth," Nano Lett. 12, 4570-4576 (2012).

${ }^{188}$ A. Mazid Munshi and H. Weman, "Advances in semiconductor nanowire growth on graphene," Phys. Status Solidi RRL 7, 713-726 (2013).

${ }^{189}$ J. M. Spurgeon et al., "Repeated epitaxial growth and transfer of arrays of patterned, vertically aligned, crystalline Si wires from a single $\mathrm{Si}(111)$ substrate," Appl. Phys. Lett. 93, 032112 (2008).

${ }^{190}$ M. Yao et al., "Tandem solar cells using GaAs nanowires on Si: Design, fabrication, and observation of voltage addition," Nano Lett. 15, 7217-7224 (2015).

${ }^{191}$ Y. Chen, O. Höhn, N. Tucher, M.-E. Pistol, and N. Anttu, "Optical analysis of a III-V-nanowire-array-on-Si dual junction solar cell," Opt. Express 25, A665 (2017)

${ }^{192}$ V. Maryasin et al., "Technological guidelines for the design of tandem III-V nanowire on Si solar cells from opto-electrical simulations," Sol. Energy Mater. Sol. Cells 172, 314-323 (2017).
${ }^{193}$ Y. Wang, Y. Zhang, D. Zhang, S. He, and X. Li, "Design high-efficiency III-V nanowire/Si two-junction solar cell," Nanoscale Res. Lett. 10, 269 (2015).

${ }^{194} \mathrm{~L}$. Wen et al., "Theoretical consideration of III-V nanowire/Si triplejunction solar cells," Nanotechnology 23, 505202 (2012).

${ }^{195}$ R. R. Lapierre, "Theoretical conversion efficiency of a two-junction III-V nanowire on Si solar cell," J. Appl. Phys. 110, 014310 (2011).

${ }^{196}$ N. Anttu, V. Dagyte, X. Zeng, G. Otnes, and M. Borgström, “Absorption and transmission of light in III-V nanowire arrays for tandem solar cell applications," Nanotechnology 28, 205203 (2017).

${ }^{197}$ M. Heurlin et al., "Axial InP nanowire tandem junction grown on a silicon substrate," Nano Lett. 11, 2028-2031 (2011).

${ }^{198}$ X. Zeng, G. Otnes, M. Heurlin, R. T. Mourão, and M. T. Borgström, "InP/GaInP nanowire tunnel diodes," Nano Res. 11, 2523 (2017).

${ }^{199}$ G. Otnes, M. Heurlin, X. Zeng, and M. T. Borgström, " $\operatorname{In}_{\mathrm{x}} \mathrm{Ga}_{1-\mathrm{x}} \mathrm{P}$ nanowire growth dynamics strongly affected by doping using diethylzinc," Nano Lett. 17, 702-707 (2017).

${ }^{200}$ O. Tizno et al., "Radial tunnel diodes based on InP/InGaAs core-shell nanowires," Appl. Phys. Lett. 110, 113501 (2017).

${ }^{201}$ M. Hocevar et al., "Growth and optical properties of axial hybrid III-V/ silicon nanowires," Nat. Commun. 3, 1266 (2012).

${ }^{202} \mathrm{~S}$. Conesa-Boj et al., "Hybrid axial and radial Si-GaAs heterostructures in nanowires," Nanoscale 5, 9633 (2013).

${ }^{203}$ S. R. Plissard et al., "From InSb nanowires to nanocubes: Looking for the sweet spot," Nano Lett. 12, 1794-1798 (2012).

${ }^{204} \mathrm{~S}$. Z. Oener et al., "Charge carrier-selective contacts for nanowire solar cells," Nat. Commun. 9, 3248 (2018).

${ }^{205}$ M. T. Borgstrom et al., "Towards nanowire tandem junction solar cells on silicon," IEEE J. Photovoltaics 8(3), 733-740 (2018). 